ARTICLE

https://doi.org/10.1038/s41467-019-10479-4

\title{
The transcription factor Slug represses p16 lnk4a and regulates murine muscle stem cell aging
}

Pei Zhu' ${ }^{1}$, Chunping Zhang ${ }^{1}$, Yongxing Gao ${ }^{2,3}$, Furen Wu ${ }^{1,3}$, Yalu Zhou ${ }^{1,3} \&$ Wen-Shu Wu ${ }^{1}$

Activation of the p16 lnk4a-associated senescence pathway during aging breaks muscle homeostasis and causes degenerative muscle disease by irreversibly dampening satellite cell (SC) self-renewal capacity. Here, we report that the zinc-finger transcription factor Slug is highly expressed in quiescent SCs of mice and functions as a direct transcriptional repressor of $p 16^{\text {Ink4a }}$. Loss of Slug promotes derepression of p16 Ink4a in SCs and accelerates the entry of SCs into a fully senescent state upon damage-induced stress. p16 Ink4a depletion partially rescues defects in Slug-deficient SCs. Furthermore, reduced Slug expression is accompanied by $16^{\text {Ink4a }}$ accumulation in aged SCs. Slug overexpression ameliorates aged muscle regeneration by enhancing SC self-renewal through active repression of p16 Ink4a transcription. Our results identify a cell-autonomous mechanism underlying functional defects of SCs at advanced age. As p16 $6^{\text {Ink4a }}$ dysregulation is the chief cause for regenerative defects of human geriatric SCs, these findings highlight Slug as a potential therapeutic target for agingassociated degenerative muscle disease.

\footnotetext{
${ }^{1}$ Department of Medicine, College of Medicine, University of Illinois at Chicago, Chicago, IL 60612, USA. ${ }^{2}$ Department of Medicine, The Johns Hopkins University School of Medicine, Baltimore, MD 21205, USA. ${ }^{3}$ These authors contributed equally: Yongxing Gao, Furen Wu, Yalu Zhou. Correspondence and requests for materials should be addressed to W.-S.W. (email: wuwenshu@uic.edu)
} 
S keletal muscle is a homeostatic tissue that is capable of making up turnover caused by daily wear-and-tear as well as regenerating upon damage. However, such homeostasis declines with aging. Sarcopenia, featured with progressive loss of muscle mass and strength, is one of the most common health issues for individuals at advanced age ${ }^{1}$. Therefore, determining the mechanisms that underlie skeletal muscle aging becomes essential for clinical therapy for the degenerative muscle disease of this kind.

To date, the key factors determining aging-associated decline of muscle regenerative capacity has remained open to debate. It is believed that proper muscle regeneration necessitates optimal extrinsic environmental supplies. Direct evidence supporting this viewpoint came from a whole muscle grafting experiment demonstrating that the regenerative ability of young and old muscles depended mainly on the age of the host ${ }^{2}$. More recent findings of increasing fibroblast growth factor (FGF) signaling in aged muscle fiber ${ }^{3}$, as well as the circulating protein growth differentiation factor 11 as a rejuvenating factor for aged skeletal muscle ${ }^{4}$ further highlighted the importance of local and systemic environment in muscle aging, respectively. In contrast, muscle stem cells (MuSCs) purified from aged mice show a substantial decline in the number of MuSCs that engraft and regenerate in recipient muscle ${ }^{5-7}$, shedding light on the role of stem cell intrinsic changes in regulation of muscle aging. Indeed, restoring the disordered signaling pathway, such as p38 $\mathrm{MAPK}^{6}$, in aged MuSCs rejuvenate MuSC function and muscle regeneration in old mice.

Regardless of extrinsic environment or cell-autonomous mechanisms, aging-associated changes lead to stem cell exhaustion in the elderly. Pax7-expressing satellite cells (SCs) function as a necessary stem cell population responsible for growth, maintenance, and regeneration of skeletal muscle ${ }^{8,9}$. Cellular senescence is an important cause of stem cell exhaustion with aging in multiple tissues. Telomere shortening, nontelomeric DNA damage and derepression of the INK4/ARF locus are all able to induce senescence $\mathrm{e}^{10-12}$. Notably, derepression of $p 16^{\text {Ink } 4 a}$ switches geriatric SCs from reversible quiescence into senescence, leading to incompetency of activation on muscle injury even in a youthful environment ${ }^{5}$. However, those cell intrinsic components regulating $p 16^{\text {Ink4a }}$ expression in SCs remain largely unknown.

In this study, we establish Slug, a member of zinc-finger transcription factor in the Slug/Snail superfamily, as a transcriptional repressor of $p 16^{I n k 4 a}$ in SCs. Akin to mice with aging, loss of Slug endows adult SCs features of pre-senescence by largely inducing $p 16^{\operatorname{Ink} 4 a}$ expression, triggering apparent regenerative defects during serial muscle damage. Importantly, reduced Slug and elevated p16 ${ }^{I n k 4 a}$ expressions in SCs simultaneously occur with chronological aging. Restoration of Slug expression is capable of rejuvenating aged SC functions. Our results highlight Slug as a key target for aging-associated degenerative muscle disease.

\section{Results}

Slug deletion causes a defect in muscle regeneration. The transcription factor Slug is expressed in a variety of normal tissues in the adult mouse ${ }^{13}$, indicating its important roles in development. In agreement with this notion, Slug knockout mice showed numerous abnormalities such as smaller body size and weight (Supplementary Fig. 1a,b). Since skeletal muscle accounts for $\sim 40 \%$ of adult body weight ${ }^{9}$, we examined if the reduced body weight in Slug-deficient mice is due to a reduction of muscle mass. Hindlimb muscles of adult Slug-mutant mice were reduced by $21-36 \%$ in weight (Supplementary Fig. 1c). To some extent, the lost muscle mass was caused by reduction in myofiber size in
Slug-deficient mice (Supplementary Fig. 1d, e and Fig. 1e). However, when normalized to body weight, none of the relative weights of these muscles were affected by the absence of Slug (Supplementary Fig. 1f). Changes in muscle mass may be resulted from changes in protein or cell turnover ${ }^{14}$. The latter reflects the balance between myonuclear accretion and loss. Proliferation and fusion of SCs increases the number of myonuclei within the muscle fibers. Therefore, we determined the effect of Slug deficiency on MuSC maintenance. Unexpectedly, Slug-deficient mice had even slightly higher fraction of SCs in non-lineage cell subpopulation (Fig. 1a). The total yield of SCs calculated as per milligram of harvested hindlimb muscle was also increased in Slug knockout mice (Fig. 1b). Such Slug ablation-induced increases in MuSC frequency and number were further confirmed by staining of $\mathrm{Pax}^{+}$nuclei on freshly prepared TA muscle cryosections (Fig. 1c, d).

SCs is essential for the maintenance and regeneration of skeletal muscle. Thus, we determined how Slug null-induced increase of SCs affects muscle regeneration upon injury. H\&E staining showed that Slug-deficient muscles regenerated as well as that of wild-types after a single injury (Fig. 1e-g). Strikingly, upon second injury, Slug-deficient muscles exhibited severely impaired regeneration when compared with wild-type counterparts (Fig. 1e-g). Collectively, these data demonstrated that Slug is essential for efficient muscle repair during continuous muscle regeneration, suggesting a key role of Slug in regulation of SC function.

SC-specific Slug loss impairs skeletal muscle regeneration. Skeletal muscle regeneration is a highly coordinated process involving the activation of various cellular and molecular responses ${ }^{15}$. We first decided to examine the expression pattern of Slug in SCs in view of their critical role in muscle regeneration. By comparing with several other muscle resident cell types including fibro-adipogenic progenitors (FAPs), pan-lymphocytes (LCs), and epithelial cells (ECs), in which the role of Slug is well characterized, we found that Slug is most highly expressed in quiescent SCs (Fig. 2a), and its expression was slightly reduced in activated SCs and markedly decreased after SCs were differentiated into myotubes (Fig. 2b).

To determine whether the impaired muscle regeneration in global Slug knockout mice is a SC-driven defect, we generated SCspecific Slug knockout mouse line using the Cre/loxP system (Fig. 2a-d and Supplementary Fig. 2). Unlike the global Slug knockout mice, Slugflfl Pax $7^{C r e /+}$ (designated as Slug ${ }^{c K O}$ ) mice showed no apparent differences with the control animals $\left(\right.$ Slugfl/ $/$ Pax $\left.{ }^{C r e /+}\right)$ in body size and weight (Fig. 2e). Furthermore, SCs from Ctrl but not SlugcKO mice displayed positivestaining for Slug protein, indicating that Slug is efficiently deleted in SCs in SlugcKO mice (Fig. 2f).

Next, we investigated the effect of SC-specific Slug deletion on the maintenance and regenerative capacity of SCs. Consistently, both the overall frequency and total yield of SCs calculated as per milligram of muscles were increased in Slug ${ }^{c K O}$ mice (Fig. $2 \mathrm{~g}, \mathrm{~h}$ ). Although individual myofiber diameters of the intact TA and cross-sectional area of TA on day 10 post single $\mathrm{BaCl}_{2}$ injury were not different between Ctrl and mutant littermates, more severely compromised muscle regeneration with a large increase of both necrotic fibers and fibrotic tissue was observed in Slug $c K O$ mice administered with double and triple muscle damages (Fig. 2i, j). The impaired secondary but not primary muscle regeneration was also detected in Slugfl/fl $\operatorname{Pax} 7^{C r e E R}$ mice, in which Slug was deleted specifically in adult SCs by administering tamoxifen prior to muscle injury (Supplementary Fig. 3). Taken together, these results demonstrated that the presence of Slug in SCs is essential 


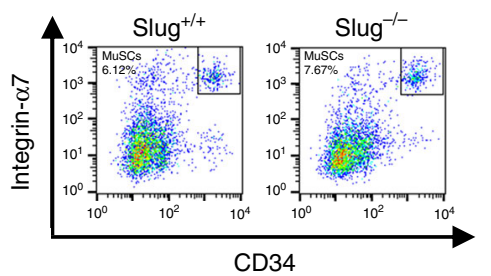

e

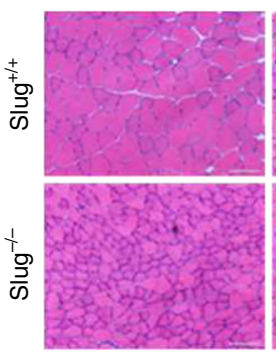

Single

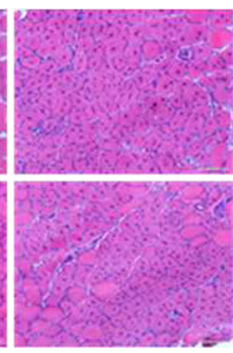

b

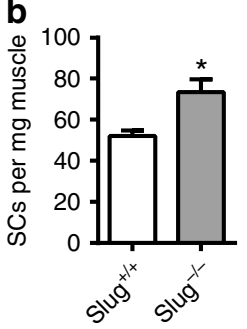

Double

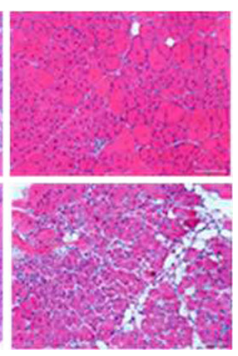

c
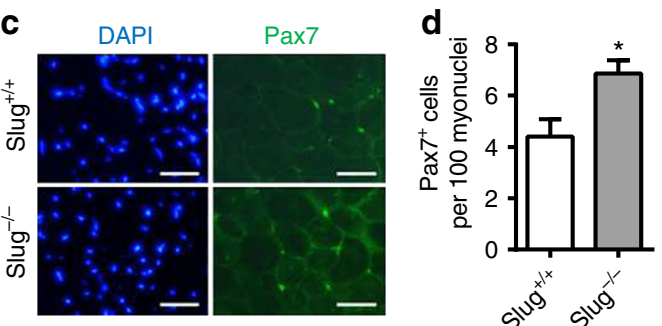

Fig. 1 Slug deficiency repairs regenerative capacity of SCs during serial muscle damage. a Representative flow cytometric analysis of the frequency of SCs (CD45-/CD11b $-/ C D 31^{-} /$Sca1 $-/$Integrin- $\alpha 7^{+} / \mathrm{CD}^{-} 4^{+}$) subpopulation in Slug ${ }^{+/+}$and Slug $/-$mice. b Yield of SCs per milligram (mg) of muscle from Slug ${ }^{+/+}$and Slug ${ }^{-/-}$mice ( $n=6$ mice per group). ${ }^{\star} p<0.05$ by student's $t$-test. c Representative IHC images of Pax $7^{+}$SCs within the intact tibialis anterior (TA) muscles of Slug $/ /+$ and Slug $/-$ mice. DAPI was used as nuclear counterstaining. Scale bar, $100 \mu \mathrm{m}$. d Quantification of Pax $7+$ SC numbers in $\mathbf{c}{ }^{\star} p<0.05$ by student's $t$-test. e H\&E staining of the intact and injured TA muscles in Slug ${ }^{+/+}$and Slug ${ }^{-/-}$mice ( $n=5$ mice per group). For single injury, TA muscles were harvested at day 10 after $\mathrm{BaCl}_{2}$ injection. For double injury, mice were recovered for 1 month after the first BaCl 2 injection at $\mathrm{TA}$ muscles. A second $\mathrm{BaCl}_{2}$ injection was administered thereafter. TA muscles were harvested 10 days after the second $\mathrm{BaCl} \mathrm{C}_{2}$ injection. Scale bar, $100 \mu \mathrm{m}$. $\mathbf{f}$ Quantification of the mean myofiber cross-sectional area (CSA, $\mu \mathrm{m}^{2}$ ) of the intact and $\mathrm{BaCl}_{2}$-injured $\mathrm{TA}$ muscles of Slug $+/+$ and Slug $/-$ mice. $\mathbf{g}$ Ratio of myofiber CSA in the intact and $\mathrm{BaCl}_{2}$-injured TA muscles between Slug ${ }^{+/+}$and Slug ${ }^{-/-}$mice. ${ }^{\star \star} p<0.01$ by student's $t$-test (n.s., not significant). All these experiments were independently repeated three times with similar results. Data are presented as mean \pm SEM. Also see Supplementary Fig. 1 . Source data are provided as a Source Data file

for SC-driven skeletal muscle regeneration, and Slug regulates muscle regeneration via its SC-specific function.

Slug-null SCs fail to replenish SC pool after activation. Maintaining SC pool size is crucial for constant muscle turnover/ injuries and ongoing repair ${ }^{16}$. We showed that muscle regeneration after first injury was normal, indicating that Slug-deleted SCs in intact muscles were functional in terms of activation and differentiation upon injury. Indeed, primary Slug ${ }^{-1-}$ SCs showed robust potential for differentiation upon in vitro induction and in vivo transplantation (Supplementary Fig. 4). However, the consequent regeneration became severely compromised in the absence of Slug (Figs. 1e, 2i and Supplementary Fig. 3e). These results prompted the question whether Slug-deficient SCs were capable of self-renewing and replenishing the stem cell pool after activation. SlugcKO displayed a sharp decrease in SC number of regenerated TA muscle when compared with Ctrl littermates after single and double injuries (Fig. 3a), indicating that lack of Slug attenuates ability of SCs to properly maintain the stem cell pool in repaired muscle after injury. This was confirmed by an observation of about three-fold decrease in SC number per 100 myonuclei in regenerated TAs of SlugcKO mice when compared with control mice (Fig. 3b, c).

Injection of tractable adult SCs into pre-injured adult muscle followed with fluorescence-activated cell sorting (FACS) analysis provides a quantitative assay for SC self-renewal ${ }^{17}$. By this assay, we analyzed the stem cell repopulation of donor-derived SCs $\left(\mathrm{GFP}^{+}\right)$ as a fraction of the total SC population from the primary recipients (Fig. 3d). Because a small portion of SCs remain cycling 1 month after transplantation ${ }^{18}$, we analyzed SCs with distinct immunophenotype $\left(\mathrm{CD} 31^{-} \mathrm{CD}_{4} 5^{-} \mathrm{Sca}{ }^{-} \mathrm{Vcam} \mathrm{I}^{+}\right)$, which consist of both quiescent and activated MuSCs ${ }^{19}$. Via this analysis, we found that the frequency of Slug ${ }^{-1}$ SCs was about three-fold lower than that of Slug $+/+$ SCs (Fig. 3e, f). Since the compromised self-renewing capability of SCs from Slug ${ }^{-/}$GFP mice may be due to embryonic absence of Slug, we induced an acute Slug knockout in SCs from Slugfl/fl Pax7-zsgreen mice by infecting with retrovirus expressing Cre recombinase (Supplementary Fig. 5a,b). Cre-expressing SCs yielded about five-fold less $\mathrm{GFP}^{+}$fraction in the total SC population from the recipients compared to that of Ctrl virusinfected SCs (Supplementary Fig. 5c,d). Consistently, by zsgreen staining we identified considerably less Cre retrovirus-infected SCs in the SC niche, beneath the basal lamina and atop myofibers $(p<$ 0.01 by student's $t$-test) (Supplementary Fig. $5 \mathrm{e}, \mathrm{f}$ ). These data indicated that deletion of Slug decreases SC self-renewing and regenerative ability.

Competitive repopulation assay has been widely used as the gold standard for testing self-renewal capacity of hematopoietic stem cells ${ }^{20}$. Here, we adopted a similar competitive repopulation assay for SCs (Fig. 3g). Technically, this assay overcomes the potential variations due to different SC niches in individual recipients and uneven injection of myotoxins and SCs at different sites. By this assay, we showed that although donor SCs from Slug ${ }^{+/+}$GFP and Slug ${ }^{-/}$GFP mice were equally mixed before transplantation, only the DNA band corresponding to wild-type alleles of Slug was predominantly amplified from the gDNA of the donor-derived SC mixtures in recipients (Fig. $3 \mathrm{~h}$ and Supplementary Fig. 6), indicating a markedly attenuated self-renewing capability in Slug $^{-/}$SCs.

To further assess the intrinsic self-renewing propensity of Slug null SCs, we performed myofiber-associated SCs culture in the presence of arabinosylcytosine (AraC) that eliminates cycling cells by inhibiting DNA synthesis. It was previously demonstrated 
a

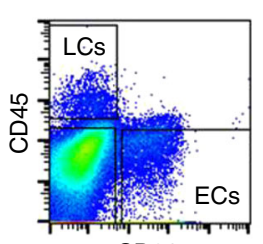

CD31

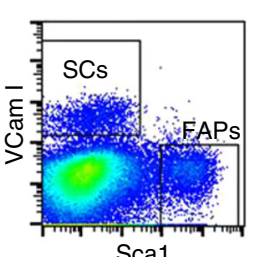

Sca1

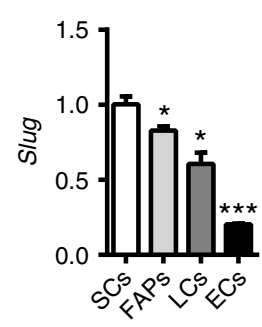

Targeting vector

Slug wilt-type allele

Targeted allele

Conditional allele

Conditionalalele

SC-specific knockout
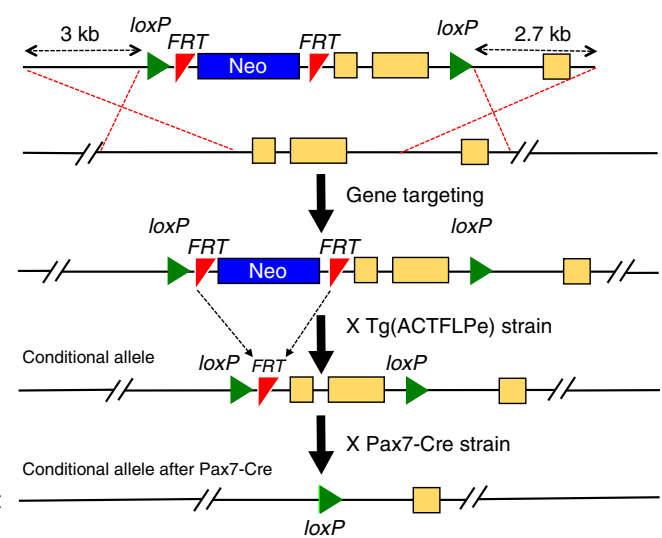

b

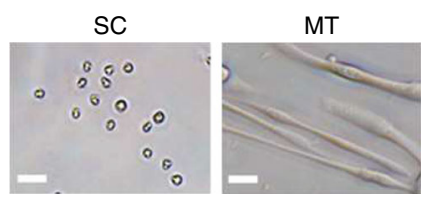

d

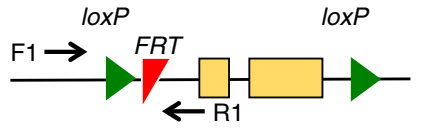

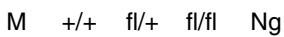

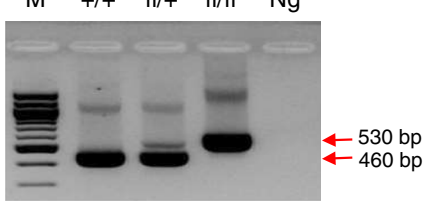

e
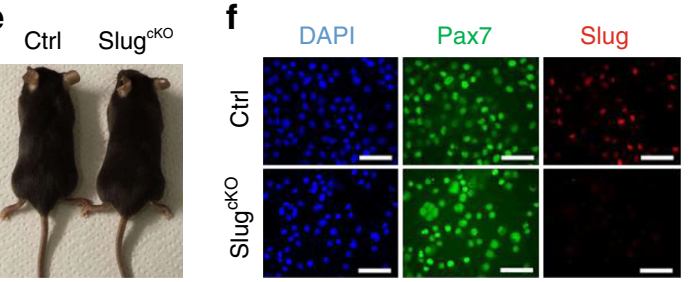

g
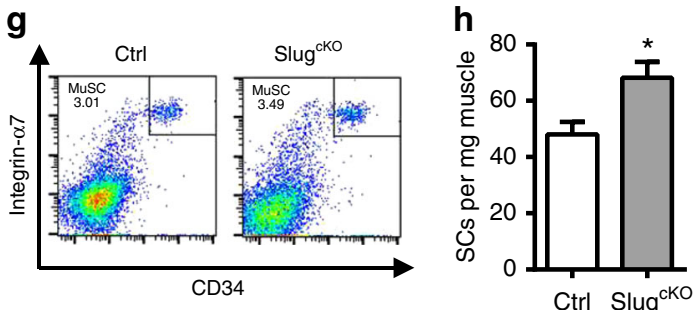

i
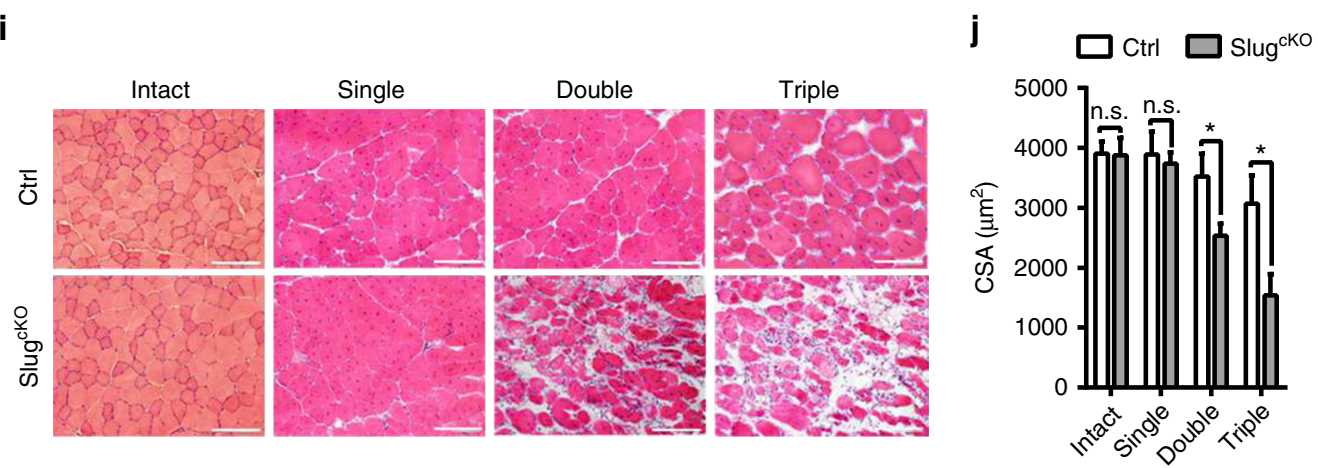

Fig. 2 SC-specific Loss of Slug Impairs Muscle Regeneration. a Slug expression in different muscle resident cells. Left, representative flow cytometric gating of SCs $\left(C D 31^{-}\right.$CD45-Scal1-Vcam I+), pan-lymphocytes $\left(L C s, C D 45^{+}\right)$, epithelial cells $\left(E C s, C D 31^{+}\right)$, and fibro-adipogenic progenitors (FAPs, $\left.\mathrm{CD}{ }^{-} \mathrm{CD} 45^{-} \mathrm{Scal}^{+}\right)$from freshly prepared skeletal muscle cells. Right, qPCR analysis of Slug expression. ${ }^{*} p<0.05,{ }^{\star *} p<0.01,{ }^{\star \star *} p<0.001$ by student's t-test. $\mathbf{b}$ Quantification of Slug expression in undifferentiated and differentiated SCs. Left, representative images of SCs and myotubes. Scale bar, $100 \mu \mathrm{m}$. Right, qPCR analysis of Slug expression. ${ }^{\star} p<0.05,{ }^{\star \star \star} p<0.001$ by student's $t$-test. QSC, quiescent satellite cell; ASC, activated satellite cells upon culture in growth medium for 3 days; MT, myotube. c Gene targeting strategy for generation of SC-specific Slug knockout mice. d Diagram of Slug-specific primer design for genotyping PCR. M. DNA marker; Ng negative control for PCR. e Comparison of adult Slugfl/flPax $7 \mathrm{Cre} /+(\mathrm{Slug}$ (KO) and Slugfl/ $+\mathrm{Pax} 7 \mathrm{Cre} /+(\mathrm{Ctrl})$. $\mathbf{f}$ Immunofluorescence staining of Slug in SCs of SlugcKO and Ctrl mice $(n=3$ mice). Scale bar, $100 \mu \mathrm{m}$. $\mathbf{g}$ Frequency of SCs in SlugcKO and Ctrl mice. Similar results were seen from three independent flow cytometric analyses. $\mathbf{h}$ Yield of SCs per mg of muscle from Ctrl and SlugcKO mice ( $n=3$ mice for each genotype). ${ }^{\star} p<0.05$ by student's $t$-test. i H\&E staining of intact and injured TA muscles in SlugcKO and Ctrl mice ( $n=5$ mice per group). For single injury, TA muscles were harvested at day 10 after $\mathrm{BaCl}_{2}$ injection. For consecutive injury, mice with primary injury were recovered for 1 month followed by a second $\mathrm{BaCl}_{2}$ injection at the same sites. TA muscles were harvested 10 days after each injury. Scale bar, $100 \mu$ m. The experiment was repeated independently for three times with similar results. $\mathbf{j}$ Quantification of the myofiber CSA $\left(\mu \mathrm{m}^{2}\right.$ ) shown in $\mathbf{i} .{ }^{*} p<0.05$ by student's $t$-test (n.s., not significant). Data are shown as mean \pm SEM of three independent replicates. Also see Supplementary Fig. 2 and 3. Source data are provided as a Source Data file 
a

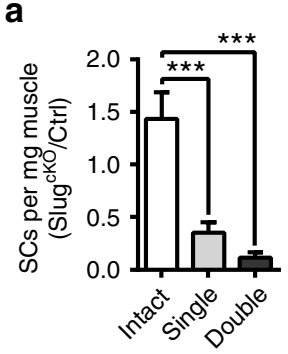

b

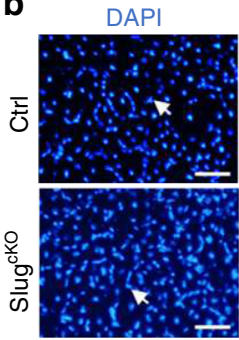

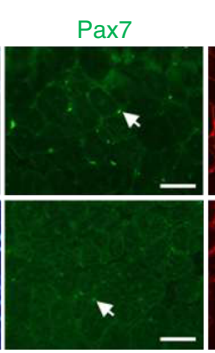

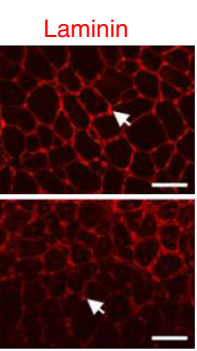

C

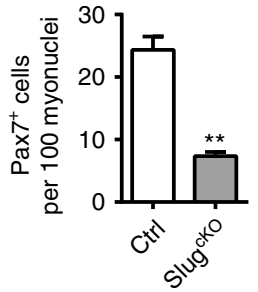

d

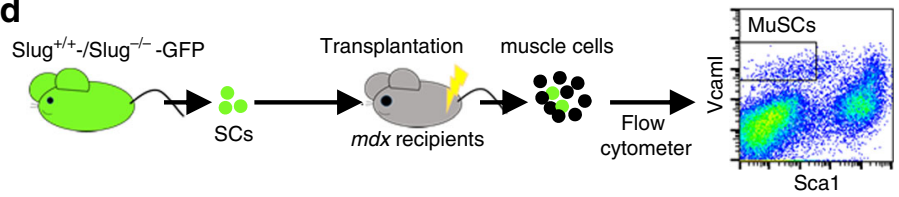

e
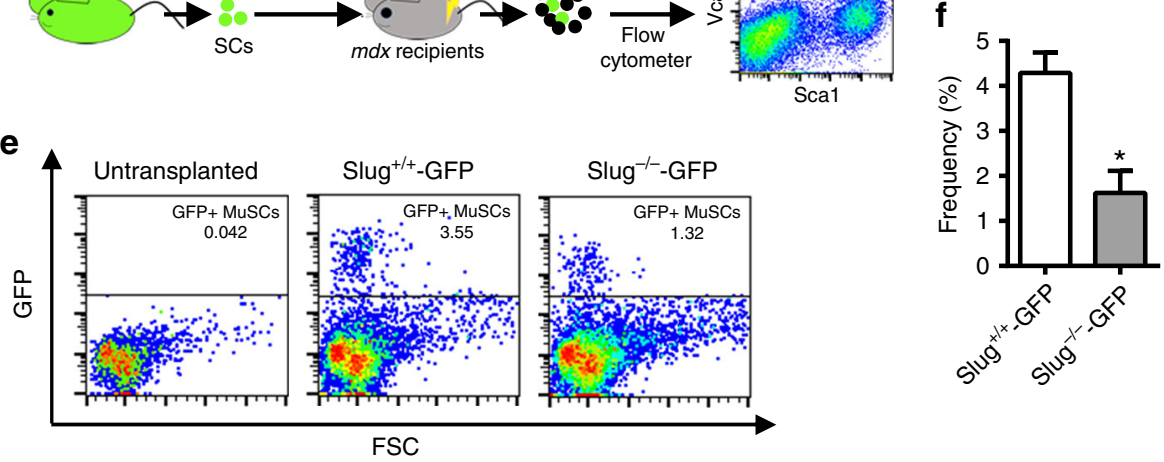

g

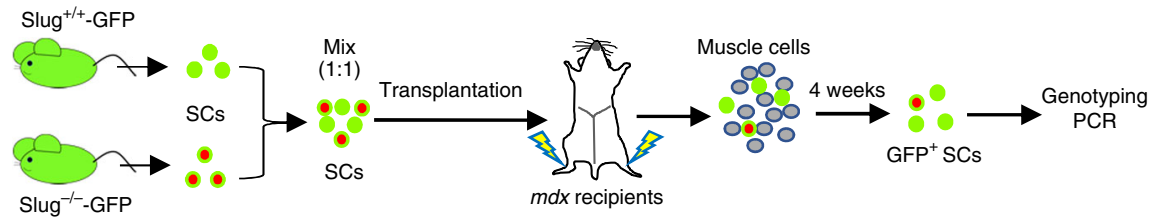

h
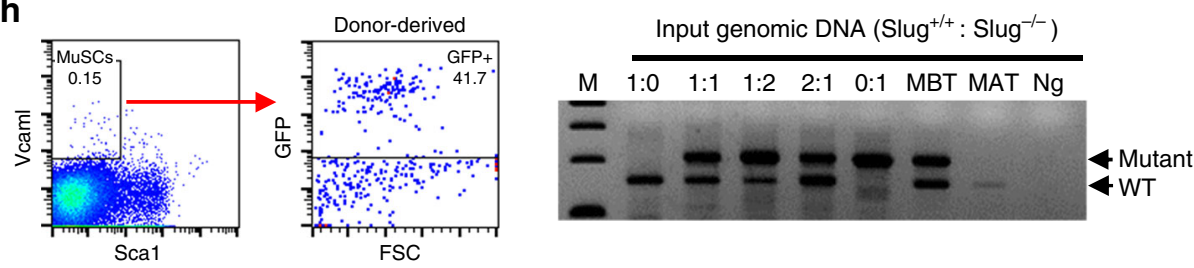

i
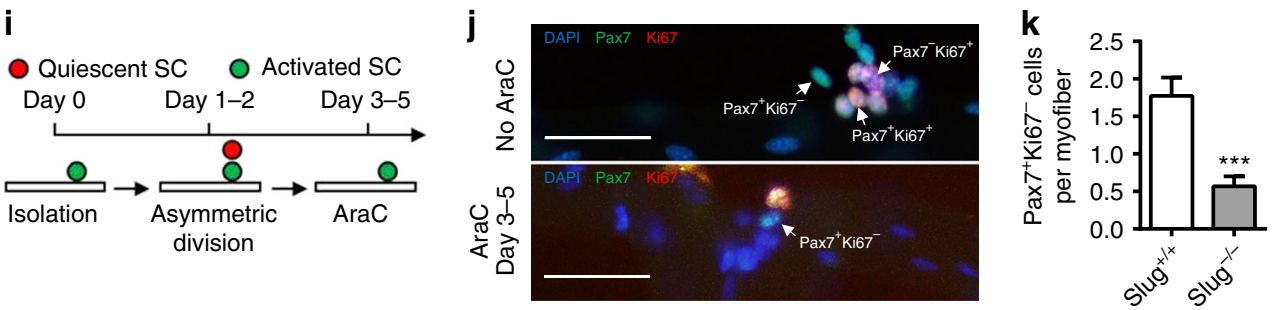

that a small population of AraC-resistant, myofiber-associated $\mathrm{Pax}^{+}$cells arose following the first SC division in culture by selfrenewing and behaved as quiescent $\mathrm{SCs} s^{6,21}$. We treated myofibers with AraC from day 3-5 after isolation (Fig. 3i) and detected a lower number of surviving $\mathrm{Pax}^{+} \mathrm{Ki}^{-}$SC daughters from Slug ${ }^{c K O}$ mice compared to those from Ctrl mice (Fig. $3 \mathrm{j}, \mathrm{k}$ ). Together, these results provide convincing evidence that Slugdeficient SCs have an intrinsic defect in self-renewal following muscle regeneration.

Slug directly represses $p 16^{\text {Ink4a }}$ transcription in SCs. To explore intrinsic factors responsible for losing self-renewal capacity of activated SCs in Slug-deficient mice, we initially retrieved and interrogated the global gene expression data (GEO accession:
GSM38236) generated from Slug-silenced primary myoblasts ${ }^{22}$. Gene ontology enrichment analysis of biological processes (GOBP) identified that Slug silencing derepressed sets of genes related to transcription regulation, cell proliferation, and skeletal muscle differentiation processes (Fig. 4a, left panel). Notably, the genes downregulated upon Slug silencing identified enrichment of genes among various categories of cellular defense responses (Fig. 4a, right panel). Interestingly, p16 ${ }^{\text {Ink } 4 \mathrm{a}}$ was listed in the most upregulated genes ranked in negative regulation of cell proliferation. It was previously reported that self-renewal capacity of aged stem cells (hematopoietic stem cells ${ }^{23}$, intestinal stem cells ${ }^{24}$, and skeletal $\mathrm{MuSCs}^{5}$ ) was attenuated. Therefore, we postulated p16 ${ }^{\text {Ink4a }}$ as a potential mediator for SC self-renewing defect caused by Slug deficiency. 
Fig. 3 SCs deficient in Slug exhibit intrinsic defects in self-renewal in vivo. a Ratio of SCs between SlugcKO and Ctrl mice under indicated conditions. ${ }^{\star \star \star} \mathrm{p}<0.001$ by student's $t$-test. $\mathbf{b} \mathrm{IHC}$ for Pax $7^{+} \mathrm{SC}$ in TAs of Slug ${ }^{c K O}$ and Ctrl mice ( $n=3$ mice per group) at day 30 post single injury. Laminin indicates the boundaries of myofibers. Scale bar, $100 \mu \mathrm{m}$. Three independent experiments were performed with similar results. c Quantification of Pax $7+\mathrm{SC}$ numbers in $\mathbf{b}{ }^{\star \star} p<0.01$ by student's $t$-test. $\mathbf{d}$ Scheme of SC transplantation. 3000 SCs of indicated genotype were transplanted into each side of pre-injured TA muscles of $m d x$ recipients $(n=6)$, respectively. Donor-derived SCs $(G F P+)$ in total recipient MuSCs $\left(\mathrm{CD}_{4} 5^{-} \mathrm{CD}^{-} 1^{-} \mathrm{Sca} 1^{-} \mathrm{Vcam} \mathrm{I}^{+}\right)$were analyzed 4 weeks after transplantation. e Representative flow cytometric analysis of the frequency of donor-derived SCs (GFP + ) within total recipient MuSCs. Similar results were seen in three independent transplantation experiments. f Percent of donor-derived SCs (GFP+) in total recipient MuSCs. ${ }^{\star} p<0.05$ by student's $t$-test. $\mathbf{g}$ Diagram of competitive MuSC repopulation assay. SCs from Slug $+/+$ GFP- and Slug ${ }^{-/-G F P-t r a n s g e n i c ~ m i c e ~ w e r e ~ e q u a l l y ~}$ mixed for genomic DNA extraction and transplantation, respectively. The remaining cells were then transplanted into pre-injured TA muscles of $m d x$ recipients. Four weeks after transplantation, GFP+ cells were sorted from total recipient MuSCs for genotyping PCR analysis. $\mathbf{h}$ Determination of relative ratio of repopulated MuSCs. Left, representative flow cytometric plotting of GFP+ cells from total recipient MuSCs. Right, PCR determining the ratio of SCs before and after transplantation. M DNA ladder, MBT mixed SCs before transplantation, MAT mixed SCs after transplantation, Ng negative control. i Scheme of AraC treatment indicating self-renewal. $\mathbf{j}$ Pax7 and Ki67 immunostaining in cultured myofibers as treated in i. Scale bar, $50 \mu m$. Similar results were seen from three independent experiments. $\mathbf{k}$ Quantification of the quiescent daughter SCs stained in $\mathbf{j}(n=3$ mice, $>20$ myofibers per condition). ${ }^{\star \star *} p<0.001$ by student's $t$-test. Data are shown as mean \pm SEM of three independent experiments. Unprocessed gel blots are provided in the Supplementary Fig. 1. Also see Supplementary Fig. 4-6. Source data are provided as a Source Data file

We then performed microarrays analysis to compare the genome-wide gene expression profiles of wild-type and Slugdeficient SCs. Unsupervised hierarchical clustering analysis separated the samples into their respective genotypes (Fig. 4b). We identified 168 differentially expressed genes, of which 108 genes were upregulated (fold change $>2, p$ value $<0.05$ ) and 60 genes were downregulated (fold change $<-2, p$ value $<0.05$ ) in Slug ${ }^{-/}$SC compared to control cells (Fig. 4b). Slug deletion enriched GO categories related to mitochondria metabolism and cell cycle regulators (Fig. 4c). To identify pathways enriched in Slug ${ }^{-1-}$ SCs, gene set enrichment analysis (GSEA) ${ }^{25}$ was performed. Notably, signaling pathways involved in cell metabolism including glycolysis, PI3K-AKT-MTOR, oxidative phosphorylation, and reactive oxygen species were induced in quiescent Slug ${ }^{-1-}$ SCs (Supplementary Fig. 7a,b), suggesting a switched metabolic reprogramming with relatively higher energyconsuming status in Slug null SCs. Meanwhile, E2F targets and G2M checkpoints signaling signatures were also enriched upon Slug deletion (Supplementary Fig. 7c,d). These gene sets enrichment data indicated that loss of Slug in SCs might disturb cell cycle progression and the balance between self-renewal and differentiation after activation.

Next, we examined expression of $p 16^{\text {Ink } 4 a}$ and $p 19^{A r f}$, the two gene products of the INK4A/ARF locus, by qPCR in SCs of adult wild-type and Slug ${ }^{-1-}$ mice, respectively. As shown in Fig. 4d, p16 ${ }^{\text {Ink4a }}$ was about two-fold higher in Slug-deficient SCs when compared with the wild-type counterparts, indicating a derepression of $p 16^{\text {Ink } 4 a}$ in the absence of Slug in resting SCs in vivo. In contrast, there was no apparent difference in p19Arf expression between the two types of SCs. Furthermore, we examined the proximal promoter region of $p 16^{I n k 4 a}$ and identified potential Slug-binding sites (E-box) ${ }^{26,27}$ (Fig. 4e). To facilitate assessing the occupancy of endogenous Slug at the promoter region of $p 16^{\text {Ink } 4 a}$ by chromatin immunoprecipitation (ChIP) assay, we performed Slug affinity tagging at its C-terminus in mouse myoblasts by CRISPR/Cas9-mediated gene tagging (Supplementary Fig. 8). ChIP-qPCR analysis displayed enriched binding in anti-Flag antibody immunoprecipitated gDNA fragments but not in antiIgG control (Fig. 4f), indicating that Slug occupies the promoter region of $p 16^{\operatorname{Ink} 4 a}$ in vivo. Such direct regulation of $p 16^{\operatorname{Ink} 4 a}$ promoter by Slug was further confirmed by $p 16^{I n k 4 a}$-driven luciferase reporter assay in SC-derived myobalsts (Supplementary Fig. 9a,b). Importantly, this E-box element is also present in human $p 16^{I N K 4 A}$ promoter (Supplementary Fig. 9c). Knockout or overexpression of $S L U G$ in primary human myoblasts significantly derepressed or suppressed $p 16^{I N K 4 A}$ transcript $(p<0.001$ by student's $t$-test) (Supplementary Fig. 9d-g), respectively, indicating a highly conserved role of Slug in regulating $p 16^{\operatorname{Ink} 4 a}$.

Of note, loss of Slug caused a more robust dysregulation of p16 ${ }^{\text {Ink } 4 a}$ in ex vivo cultured myoblasts ${ }^{22}$ compared to resting SCs in vivo. Normally, $p 16^{\operatorname{In} k 4 a}$ expression is elevated during aging and replicative senescence ${ }^{28}$. Myoblasts are a type of proliferating myogenic progenitor cells derived from quiescent SCs under stimulating conditions. Therefore, we asked whether derepression of p16 ${ }^{\text {Ink4a }}$ in Slug-deficient SCs would be exacerbated under stress conditions, including ex vivo culture and muscle damage. Strikingly, $p 16^{\text {Ink } 4 a}$ expression increased by over 8 -fold in ex vivo cultured Slug ${ }^{-1-}$ SCs and five-fold in in vivo injury-activated Slug ${ }^{-/}$SCs, respectively, when compared with their corresponding wild-type controls (Fig. $4 \mathrm{~g}$ ) Notably, derepression of $p 16^{\text {Ink } 4 a}$ in cultured myoblasts was accompanied with graduate decline of Slug expression (Supplementary Fig. 10).

In spite of the increased $p 16^{\text {Ink4a }}$ transcription, primary muscle regeneration was normal in Slug-deficient mice. This is a departure from what was reported in geriatric mice model, i.e., that resting SCs fail to activate and expand to regenerate the muscle on injury due to derepression of $p 16^{\text {Ink } 4 a 5}$. We suspected that $\mathrm{p} 16^{\text {Ink4a }}$ mRNA but not protein was increased in resting Slug-deficient SCs, since $p 16^{\text {Ink } 4 a}$ mRNA could be induced to decay by an RNA-binding protein in early passage of fibroblasts but accumulated at protein level during late-passage of culture ${ }^{28}$. Indeed, $\mathrm{p} 16^{\text {Ink4a }}$ protein was undetectable in undamaged muscle cryosections from both Ctrl and SlugcKO mice (Supplementary Fig. 11). Instead, there was an apparent co-expression of p16 Ink4a with Pax7 in muscle tissue harvested from SlugcKO mice 30-day post injury (Fig. $4 \mathrm{~h}, \mathrm{i})$. Taken as whole, these results suggested that Slug deficiency leads to an increase in $p 16^{\text {Ink }} 4 a$ transcription in SCs, and replicative stress signaling triggered by SC activation and proliferation concurrently increases the stability of $\mathrm{p} 16^{\text {Ink } 4 \mathrm{a}}$ protein.

Slug loss promotes senescence in SCs during proliferation. In terms of the elevated $\mathrm{p} 16^{\mathrm{Ink} 4 \mathrm{a}}$ protein in activated SCs in regenerating skeletal muscles of Slug-deficient mice, we hypothesized that Slug-ablated SCs acquired features of cellular senescence at late stages of regeneration during the transition of SCs from activation to quiescence or differentiation. To test this notion, we first used an in vitro reserve cell culture system ${ }^{29}$. As shown in Fig. 5a, reserve cells from wild-type mice robustly proliferated while cells from Slug ${ }^{-/}$mice were only sporadically distributed by day 7 of subculture (Fig. 5a). Compared to their wild-type control cells, significantly higher proportion of progeny from 


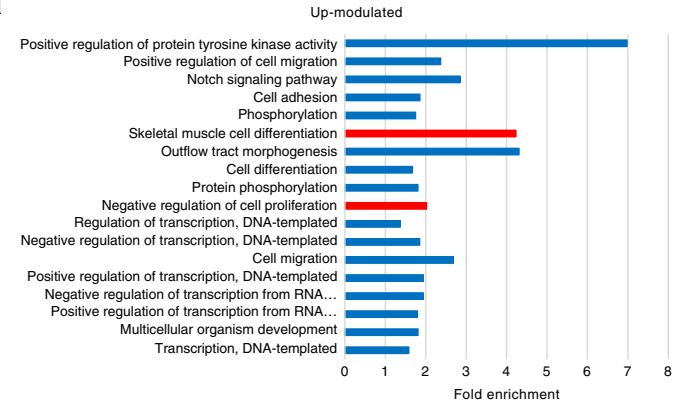

b

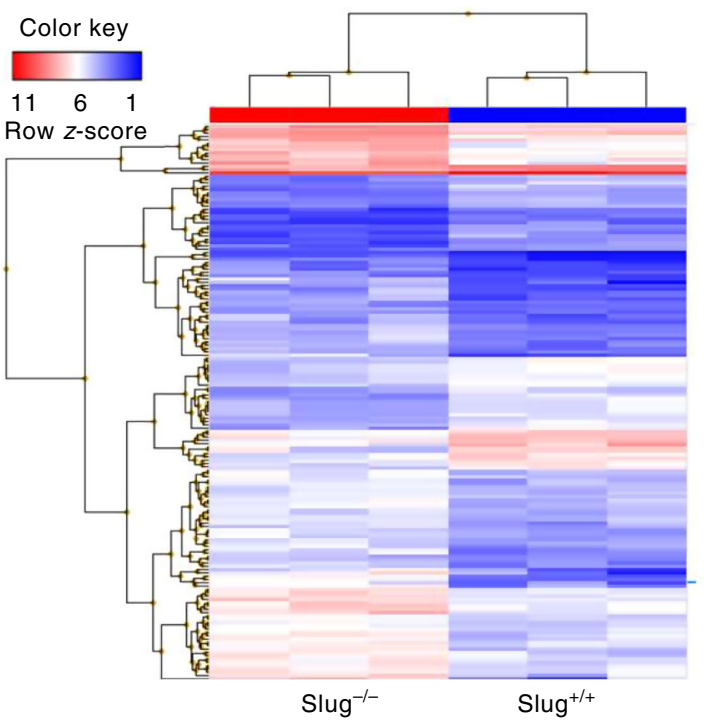

d

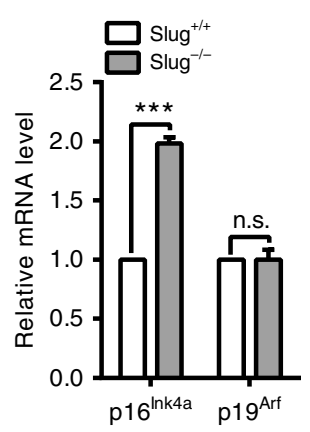

e

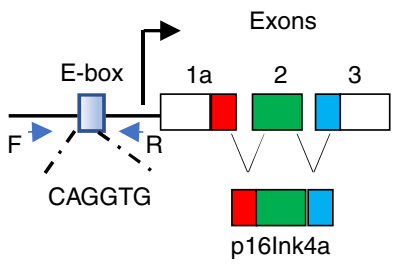

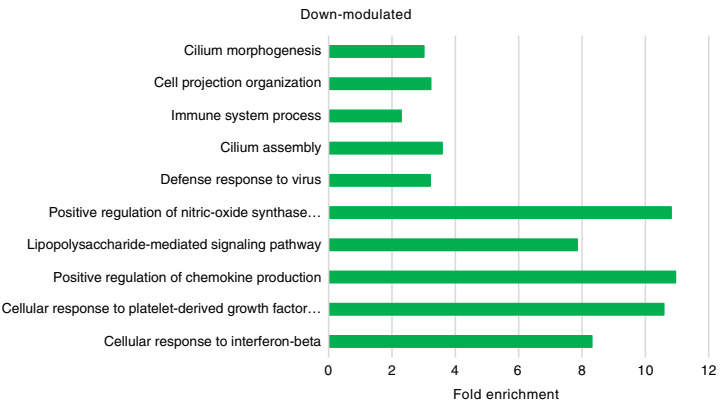

C
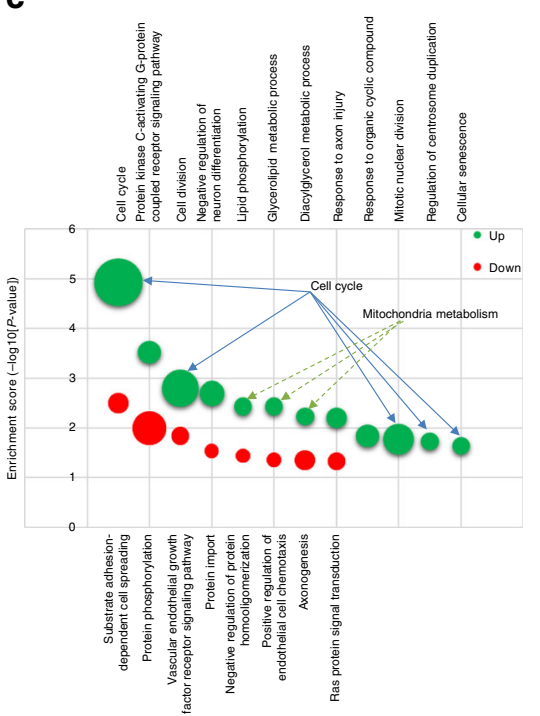

f

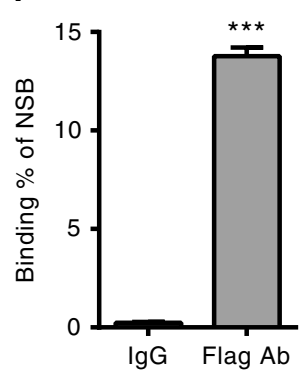

g

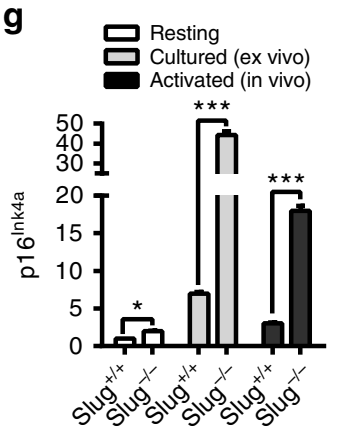

h

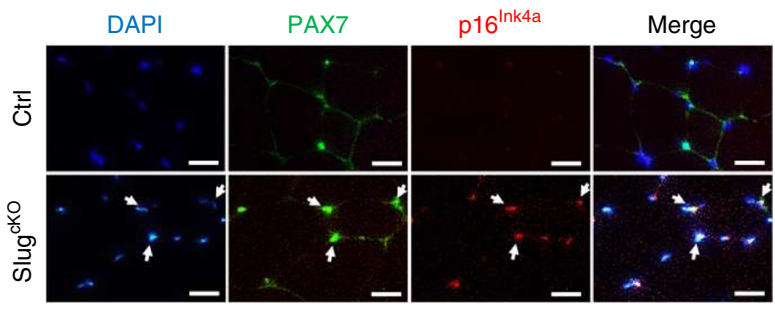

i

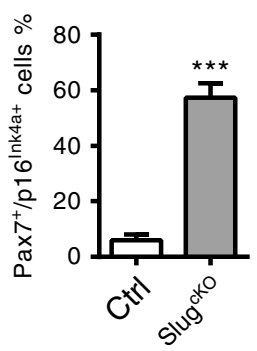

Slug ${ }^{-/-}$reserve cells was positive for $\mathrm{p} 16^{\text {Ink4a }}$ staining $(p<0.001$ by student's $t$-test) (Supplementary Fig. 12). SA- $\beta$-Gal staining showed that over $50 \%$ of Slug-deficient reserve cells-expanded cells was SA- $\beta-\mathrm{Gal}^{+}$, a marker of senescence, whereas few SA- $\beta-\mathrm{Gal}^{+}$ cells were detected in the control group (Fig. 5a, b).
Population doubling level (PDL) is an intrinsic measurement of the age of the particular culture of a cell line. In culture, an untransformed cell line has a finite life span expressed in the number of cumulative population doublings that can be achieved. To assess the accelerated senescence in Slug-deficient SCs under 
Fig. 4 p16 $6^{\operatorname{lnk} 4 a}$ is a direct target gene of Slug in SCs. a GOBP analysis of genes modulated in Slug-silenced myoblasts (GEO accession: GSE38236). The term of negative regulation of cell proliferation being highly relevant to observed self-renewal defect of Slug-deficient SCs in our study was highlighted in red. b Hierarchical clustering and heatmap representation of 168 differentially expressed genes separating Slug ${ }^{+/+}$and Slug ${ }^{-/-}$SCs. Color pattern represents row Z-score. c Bubble chart showing results of GOBP analysis. Bubble size indicated number of genes associated with each term. GOBP ranked by fold enrichment score associated with upmodulated (green bubbles) and downregulated (red bubbles) signatures were selected for significance by using a false discovery rate cutoff of 5\%. d qPCR analysis of $p 16^{\operatorname{lnk} 4 a}$ and p19Arf in primary SCs from Slug ${ }^{+/+}$and Slug ${ }^{-/-}$mice $(n=3)$. n.s. not significant; ${ }^{\star \star \star} p<0.001$ by student's $t$-test. e Schematic diagram of the INK4a/ARF. The consensus Slug-binding site (E-box) is located in its promoter. $\mathbf{f}$ ChIP analysis of Slug occupancy at the p16 Ink4a promotor. Primers targeting the $3^{\prime}$ untranslated region (without E-box elements) was used as a negative control for the ChIP assay. Relative binding affinity of Slug on the putative E-box element was quantified relative to the non-specific binding. ${ }^{\star \star \star} p<0.001$ by student's $t$-test. g Quantification of $p 16^{\operatorname{lnk} 4 a}$ in quiescent, ex-vivo culture- and in vivo injury-activated SCs from Slug ${ }^{+/+}$and Slug ${ }^{-/-}$mice. For activation ex-vivo, SCs were cultured for 7 days; while for activation in vivo, SCs were harvested at day 10 after injury. ${ }^{\star} p<0.05,{ }^{\star \star \star} p<0.001$ by student's $t$-test. $\mathbf{h} I H C$ for $p 16^{\operatorname{lnk} 4 a}$ and Pax7 protein in SCs within the TA muscles of SlugcKO and Ctrl mice at day 30 post injury ( $n=3$ mice per genotype). Scale bar, $100 \mu \mathrm{m}$. i Percentage of Pax 7 and p16 Ink4a double positive SCs in $\mathbf{h} .{ }^{\star \star \star} p<0.001$ by student's $t$-test. The experiments $\mathbf{g}$-i were independently repeated three times with similar results. Data are shown as mean \pm SEM of three independent experiments. Also see Supplementary Fig. 7-11. Source data are provided as a Source Data file

proliferative pressure in vitro, we determined the relative growth rates of control and Slug-mutant SCs by calculating the cumulative PDL. As shown in Fig. 5c, the growth rate of Slugdeficient SCs was clearly retarded at early passage 3 when wildtype SCs remained for exponential growth. SA- $\beta-$ Gal staining demonstrated that about $40 \%$ of Slug-ablated myoblasts show strong positive SA- $\beta$-Gal staining while less than $5 \%$ of control cells were SA- $\beta-\mathrm{Gal}^{+}$on passage 3 (Fig. $5 \mathrm{~d}$, e), indicating the existence of replicative senescence and growth retardation in early-passaged Slug-deficient myoblasts.

Next, we investigated whether cellular senescence in SCs would occur when Slug is specifically deleted in Pax $7^{+}$SCs during regeneration in vivo. Young adult mice were used in this study to exclude the process of regeneration from aging in geriatric mice 5 . We performed SA- $\beta$-Gal staining on cryosections of TA muscles from Ctrl and SlugcKO mice on day 10 post first injury when necrotic fibers were replaced by central-nucleated regenerated myofibers (Fig. $5 \mathrm{f}$ ). Notably, despite the sporadic SA- $\beta-\mathrm{Gal}^{+}$cells in control TA muscle sections, there was a four-fold increase in the number of SA- $\beta-\mathrm{Gal}^{+}$cells in SlugcKO TA (Fig. $5 \mathrm{f}, \mathrm{g}$ ). Most of the SA- $\beta-\mathrm{Gal}^{+}$cells were located beneath basal lamina and outside myofiber plasma membrane, a classical SC anatomical location (Fig. 5f). IHC results further demonstrated that these SA$\beta$-Gal ${ }^{+}$cells were also Pax7-positive. As expected, SA- $\beta$-Gal ${ }^{+} \mathrm{Pax}^{+}$cells were not in cycling $\left(\mathrm{Ki} 67^{-}\right)$, suggesting the status of cellular senescence. In contrast, SCs with negative SA- $\beta-$ Gal staining $\left(\mathrm{SA}-\beta-\mathrm{Gal}^{-} / \mathrm{Pax} 7^{+}\right)$were on the occasion of proliferating $(\mathrm{Ki67}+)$. In agreement with this notion, the majority of SCs in SlugcKO mice failed to re-activate as indicated by markedly lowered percentage of $\mathrm{Ki}^{+}{ }^{+}$SCs by 2.5 -day post second $\mathrm{BaCl}_{2}$ injury compared to Ctrl mice (Fig. 5i, j). Together, using multiple in vitro and in vivo assays we proved that lack of Slug facilitated entry of SCs into cellular senescence under proliferative pressure.

p16 ${ }^{\text {Ink4a }}$ loss restores impaired self-renewal of Slug ${ }^{-/-}$SCs. We demonstrated elevated $\mathrm{p} 16^{\text {Ink } 4 \mathrm{a}}$ protein and acquired senescence feature in activated Slug-deficient SCs. At this point, a key question was whether p16 $16^{\text {Ink4a }}$ is causally involved in Slug loss-induced defects in SC self-renewing capacity and muscle regeneration. To answer this question, we assessed muscle regeneration in Slug ${ }^{+/+}$ $p 16^{+/+}$, Slug $g^{-/-} p 16^{+/+}$, and Slug ${ }^{-/-} p 16^{-/-}$mice following serial damages. By H\&E staining, we showed that although muscle regeneration was severely compromised in TAs of $\operatorname{Slug}^{-/}{ }^{-} \mathrm{p} 16^{+/+}$ mice following double or triple injuries, muscle repair was greatly improved in Slug ${ }^{-/}$p16 $16^{-1-}$ mice after injuries (Fig. 6a, b).

Next, we assessed how removal of p16 Ink4a would affect the self-renewing ability of Slug-ablated SCs by the quantitative repopulating assay (Fig. 6c). As expected, the frequency of Slug ${ }^{-/-} p 16^{+/+}$donor-derived SCs was low ( 1.96\%) (Fig. 6d, e). However, the frequency of Slug ${ }^{-1-} p 16^{-1-}$ donor-derived SCs was increased by two-fold in the recipients (Fig. 6d, e). Mechanistically, enhanced repopulation of Slug $g^{-/} p 16^{-/-}$SC in recipient muscles after deletion of $p 16^{\text {Ink }} 4 a$ might be partially ascribed to reduced senescence under proliferative stress, as $\mathrm{Slug}^{-/} p 16^{-/-}$ SC-derived myoblasts displayed little or no SA- $\beta$-Gal staining at ex vivo passage 3 when majority of Slug ${ }^{-/-}$cells were SA- $\beta$-Gal positive (Fig. 6f, g). In summary, p16 Ink4a is a crucial mediator for the defects in SC self-renewal and muscle regeneration caused by Slug deficiency.

Slug overexpression restores self-renewal of aged SCs. Aging alters stem cell function in terms of the capacities of self-renewal, proper activation and/or proliferation as well as lineage commitment. Aged SCs were characterized by self-renewal $\operatorname{defect}^{6,7,16}$, inclined commitment of differentiation in low mitogen ex vivo culture condition ${ }^{3,6}$, and susceptibility to senescence upon mitogen exposure ${ }^{7,30}$. These aging-associated phenotypes resemble to some extent behaviors of Slug-deficient SCs in current study. Such similarities drew our attention to the role of Slug in SC aging. By interrogating the transcriptomes of young and aged SCs from different groups of mice ${ }^{4,31}$, we observed an ageassociated downregulation of Slug in aged SCs (Fig. 7a). This is further confirmed in SCs from young and aged mice by PCR analysis (Fig. $7 b-d$ ).

We demonstrated that there was an increased bioenergetic requirement in Slug null SCs (Supplementary Fig. 7a). Interestingly, GSEA revealed a similarly altered metabolic reprogramming in old SCs (GSE81096), which was evidenced by activation of genes ranked in glycolysis and mitochondrial metabolism in MTORC1, oxidative phosphorylation, PI3K-Akt-MTOR, and reactive oxygen species signaling pathways (Supplementary Fig. 13a). Of note, 71 genes were overlapped in these metabolic pathways of both Slug-deleted and aged SCs (Supplementary Fig. 13b). In addition, E2F targets and G2M checkpoints signatures being activated in $\mathrm{Slug}^{-1}$ SCs were also enriched in old SCs in comparison to the young SCs (Supplementary Fig. 13c). Of those cell cycle regulators being upregulated in Slug ${ }^{-1-}$ SCs, 29 of which were activated in aged SCs as well (Supplementary Fig. 13d). Together, these data indicated that Slug insufficiency might be an important factor in causing multiple regenerative defects in aged SCs.

It has been reported that replicative senescence can play a role in the regenerative defects during normal skeletal muscle aging ${ }^{32}$ and replicative senescence may be caused by $16^{\text {Ink4a }}$ stress pathway ${ }^{33}$. Next, we applied ex vivo cultured SCs as a model to 
a

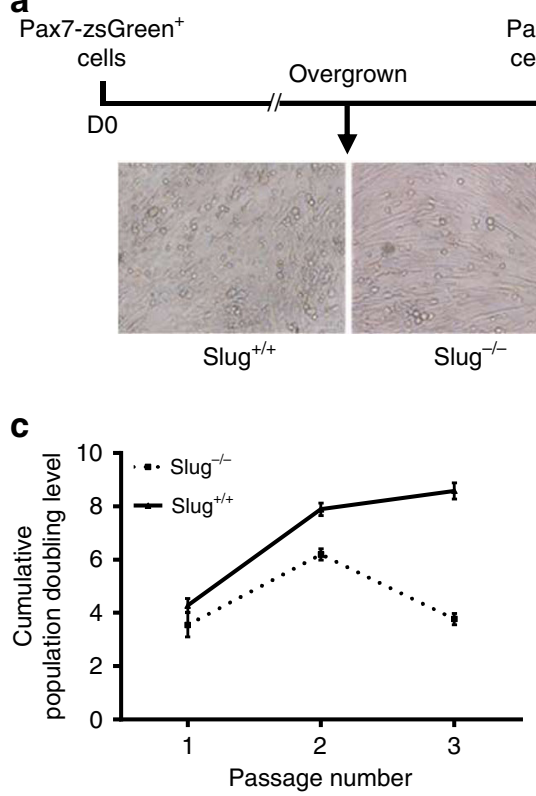

Pax7-zsGreen ${ }^{+}$ cells re-plating

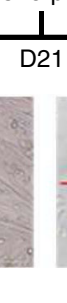

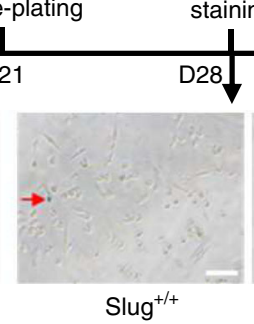

d

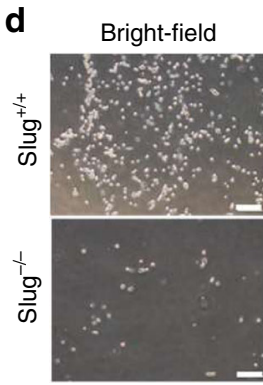

b

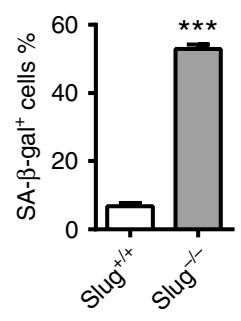

f

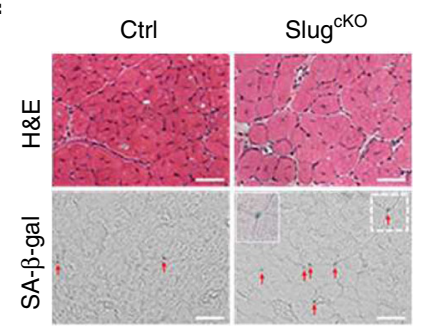

i

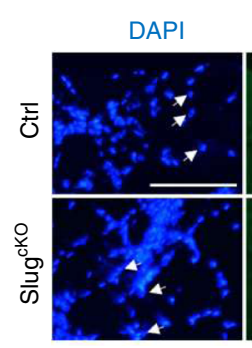

g

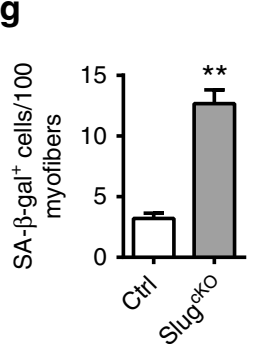

h SA- $\beta$-gal

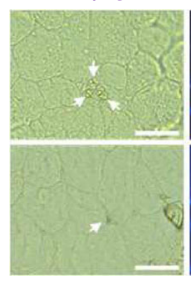

SA- $\beta$-gal

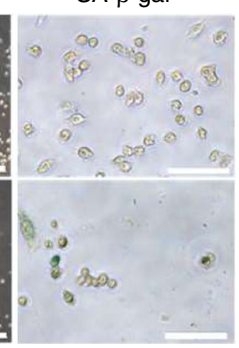

e

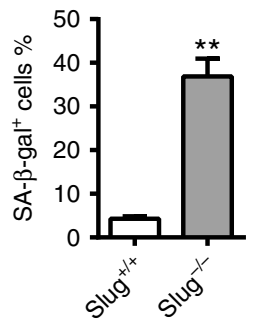

DAPI

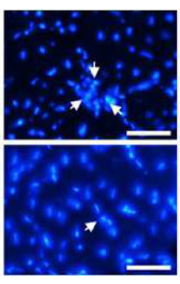

Pax7

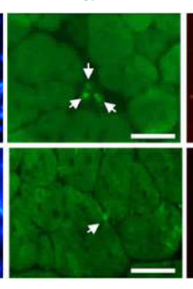

Ki67

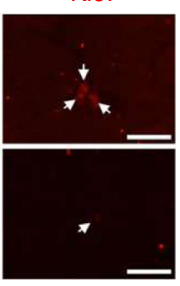

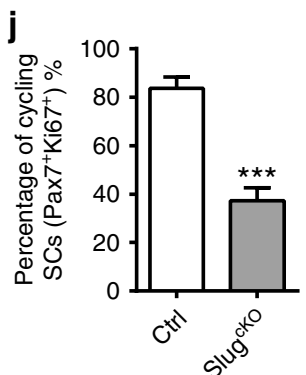

Fig. 5 Slug-deficient SCs acquire senescence properties during self-renewal. a SA- $\beta$-Gal staining. SCs of indicated genotype were differentiated for 21 days. 5000 zsGreen $^{+}$reserve cells were sub-cultured for another 7 days and subject to SA- $\beta$-Gal staining. Red arrows indicate SA- $\beta$-Gal ${ }^{+}$cells. Scale bar, 100 $\mu \mathrm{m}$. $\mathbf{b}$ Quantification of the percentage of SA- $\beta$-Gal ${ }^{+}$cells stained in $\mathbf{a} .{ }^{\star \star \star} p<0.001$ by student's $t$-test. c Cumulative population doubling level (CPDL) obtained in cultures of primary SC-derived myoblasts from Slug ${ }^{+/+}$and Slug $/-$mice. d Photographs (left) and SA- $\beta$-Gal staining (right) of SC-derived myoblasts on day 7 of culture at passage 3. Scale bar, $200 \mu \mathrm{m}$ (left); $100 \mu \mathrm{m}$ (right). e Percentage of the SA- $\beta$-Gal ${ }^{+}$cells in d. ${ }^{\star \star} p<0.01$ by student's $t$-test. f Representative images of H\&E (upper) and SA- $\beta$-Gal staining (lower) on transverse TA muscle cryosections. TA muscles from SlugcKO and Ctrl mice ( $n=3$ per genotype) were harvested on day 10 post injury and subject to H\&E and SA- $\beta$-Gal staining. Red arrows indicated SA- $\beta-G a l+c e l l s$. The window (lower panel, right picture) represents high magnification of dotted boxed area. Scale bar, $50 \mu \mathrm{m}$. $\mathbf{g}$ Quantification of the $\mathrm{SA}-\beta-\mathrm{Gal}{ }^{+}$cell numbers stained in f. ${ }^{\star *} p<0.01$ by student's $t$-test. $\mathbf{h}$ Senescent SCs in injured mice of SlugcKO and Ctrl mice. SA- $\beta$-Gal staining was combined with IHC staining against Pax7 and Ki67 on TA muscle cryosections being treated as described in f. Scale bar, $50 \mu \mathrm{m}$. i IHC staining for Pax7 and Ki67 in TA muscle sections harvested at day 2.5 post the second $\mathrm{BaCl}_{2}$ injury ( $n=3$ mice per group). Arrows indicated SCs in the section. Scale bar, $100 \mu \mathrm{m}$. $\mathbf{j}$ Percentage of cycling $\left(\mathrm{Pax} 7^{+} \mathrm{Ki} 67^{+}\right.$) SCs stained in $\mathbf{i} .{ }^{\star \star} p<0.001$ by student's $t$-test. Data are presented as mean \pm SEM of three independent experiments. Also see Supplementary Fig. 12. Source data are provided as a Source Data file

address whether forced expression of Slug could restore the intrinsic regenerative and self-renewing capacities of the aged SCs (Fig. 7e). Similar to what found in the geriatric SCs (Fig. 7c), a sharp increase in $p 16^{\text {Ink4a }}$ expression was found in cultured SCs (Fig. 7f). Forced expression of Slug significantly suppressed stressinduced $p 16^{\text {Ink } 4 a}$ ( $p<0.001$ by student's $t$-test) (Fig. 7f). Furthermore, we demonstrated that Slug-overexpressing myoblasts gave rise to a substantially larger fraction of the total MuSC population in $m d x$ recipients than myoblasts transduced with the control retrovirus (Fig. $7 \mathrm{~g}, \mathrm{~h}$ ). In addition to the self-renewal capacity, Slug overexpression also largely restored regenerative capability of SCs that underwent passaging and subculturing for weeks, as evidenced by an increase in the number of dystrophin-positive myofibers in $m d x$ recipient (Fig. $7 \mathrm{i}, \mathrm{j}$ ). 

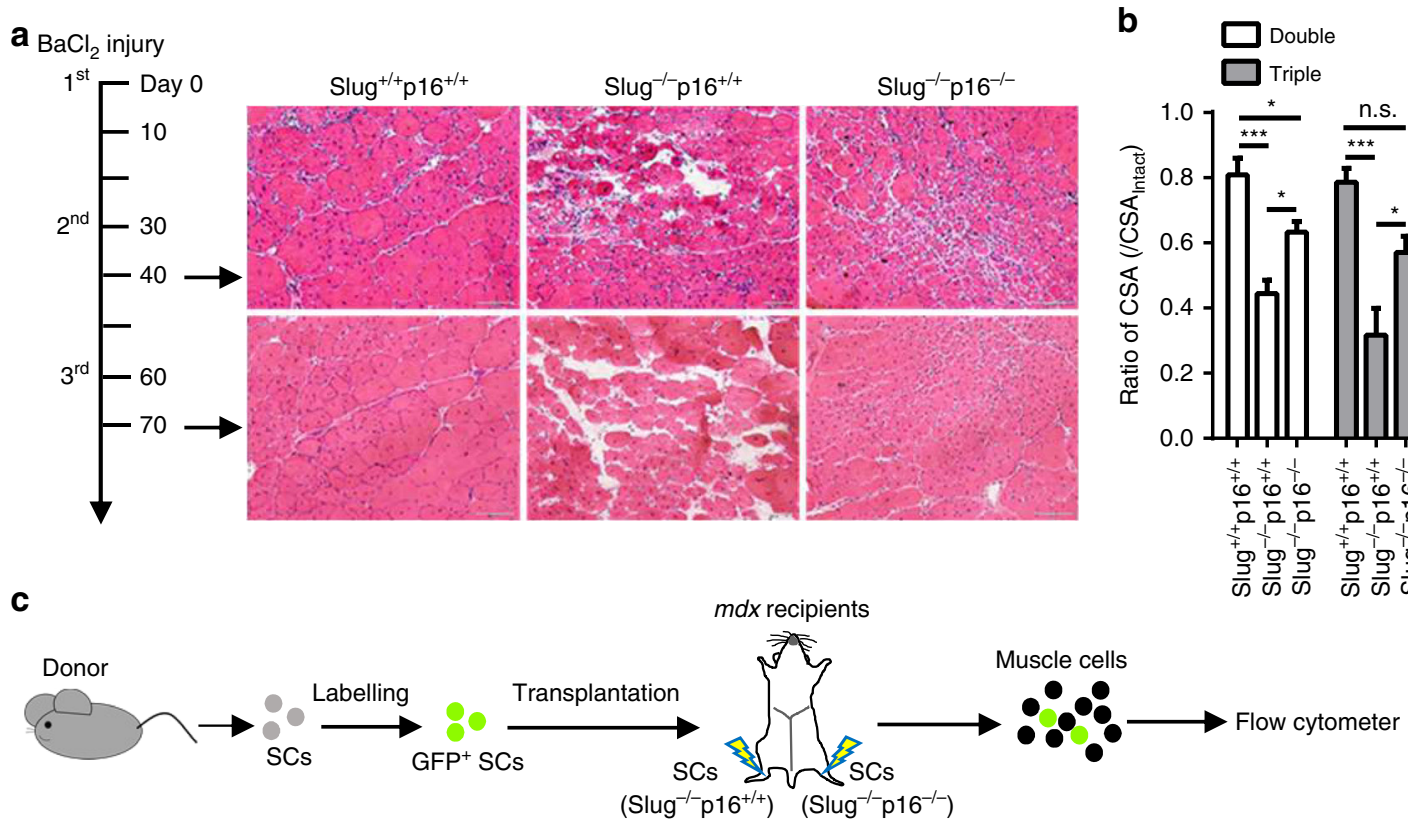

d
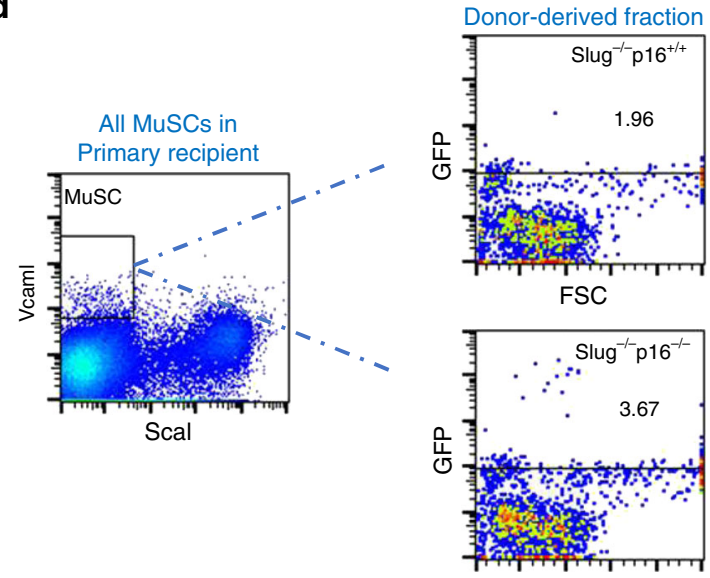

FSC

f
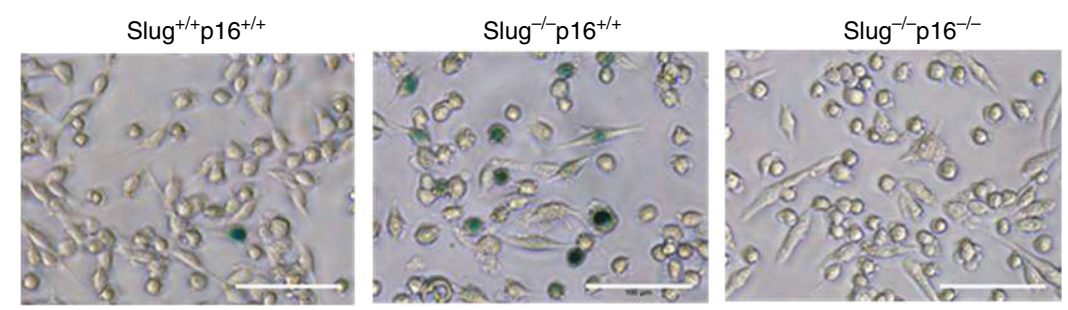

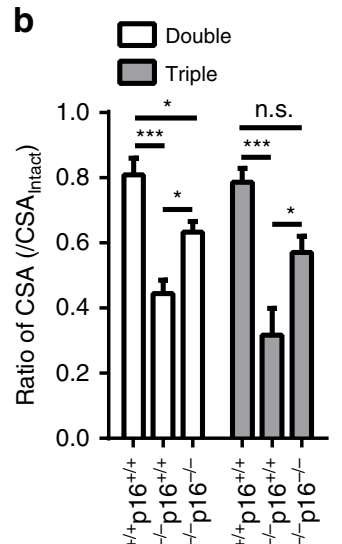

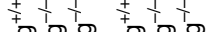
के के के के $\left(\right.$ Slug $\left.^{-/} \mathrm{p} 16^{+/+}\right) \quad\left(\right.$ Slug $\left.^{-/-} \mathrm{p} 16^{-/-}\right)$

e
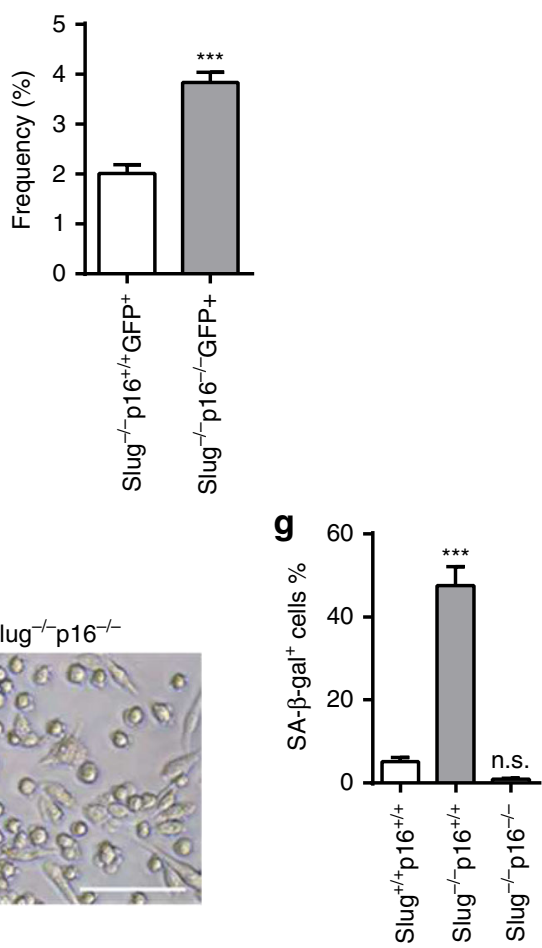

Fig. 6 Removal of p16 Ink4a partially rescues regeneration and self-renewal of Slug-deficient SCs. a H\&E staining of the double and triple BaCl ${ }_{2}$-injured TA

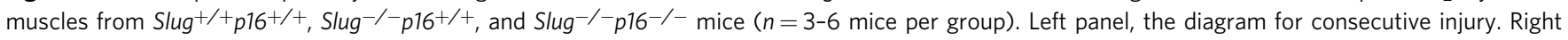
panel, representative images of H\&E staining of muscle tissue sections from injured mice. Scale bar, $100 \mu$ m. $\mathbf{b}$ Ratio of myofiber CSA in the intact and $\mathrm{BaCl}_{2}$-injured TA muscles between Slug ${ }^{+/+}$p $16^{+/+}$, Slug ${ }^{-/-}$p16 $6^{+/+}$, and Slug ${ }^{-/-}$p $16^{-/-}$mice. ${ }^{*} p<0.05,{ }^{\star \star *} p<0.001$ by one-way ANOVA; n.s. not significant. c Scheme of SC transplantation. SCs isolated from Slug ${ }^{-/-} p 16^{+/+}$and Slug ${ }^{-/-} p 16^{-/-}$mice were infected with GFP-expressing retroviruses, and injected into either side of the $\mathrm{BaCl}_{2}$-pre-injured TA muscle of $m d x$ recipient, respectively. Total mononucleated muscle cells were isolated separately from either TA muscle 4 weeks after transplantation, and subjected to flow cytometric analysis for the fraction of donor-derived SCs (GFP+) in MuSC $\left(\mathrm{CD} 45^{-} \mathrm{CD}^{-} 1^{-} \mathrm{Sca} 1^{-} \mathrm{Vcam} \mathrm{I}^{+}\right.$) subpopulation of recipient mice. $\mathbf{d}$ Representative flow cytometry plots showing the frequency of donor-derived SCs (GFP + ) within the total recipient MuSC (CD45-CD31-Sca1- ${ }^{-}$cam I+) subpopulation in mononucleated TA muscle cells. e Percent of donor-derived cells (GFP + ) in total MuSC (CD45-CD31-Sca1 $\left.{ }^{-} \mathrm{Vcam} \mathrm{I}^{+}\right)$subpopulation of mononucleated TA muscle cells from mdx recipients $(n=4-6)$. ${ }^{\star \star \star} p<0.001$ by student's $t$-test. $\mathbf{f}$ Representative SA- $\beta$-Gal staining of cultured primary SC-derived myoblasts. $10^{4}$ SCs of indicated genotype were plated in Matrigel-coated wells of 24-well plate, and passaged weekly. Bright-field imaging and SA- $\beta$-Gal staining were performed in cells on day 7 of culture at passage 3 . Scale bar, $100 \mu$ m. g Percentage of the SA- $\beta$-Gal ${ }^{+}$cells in serially passaged myoblasts on day 7 of culture at passage $3 .{ }^{\star \star \star} p<0.01$; by student's $t$-test. n.s. not significant. Data are shown as mean \pm SEM of three independent replicates. Source data are provided as a Source Data file 
a

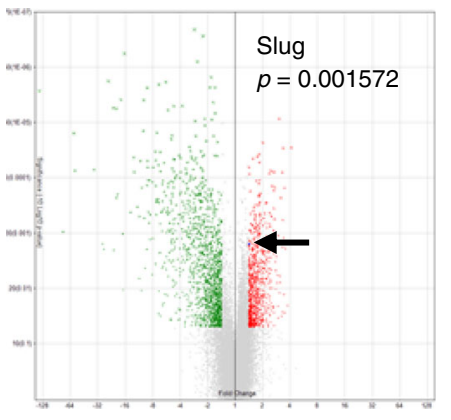

Sinha et al. 2014

(Freshly isolated young vs aged SCs) Method: CD45 ${ }^{-}$Sca- $1^{-}{\text {CD } 11 b^{-} \text {CXCR4 }^{+} \beta 1 \text {-Integrin }}^{+}$

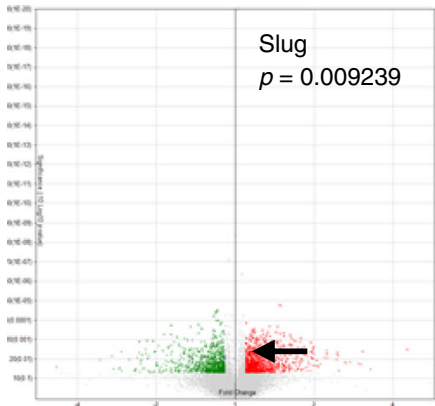

Oh et al. 2016

(Freshly isolated young vs old SCs)

Method: CD45 ${ }^{-}$Sca- $1^{-}$CD11 $^{-}$Ter119- CXCR4 ${ }^{+} \beta 1$-Integrin ${ }^{+}$ b

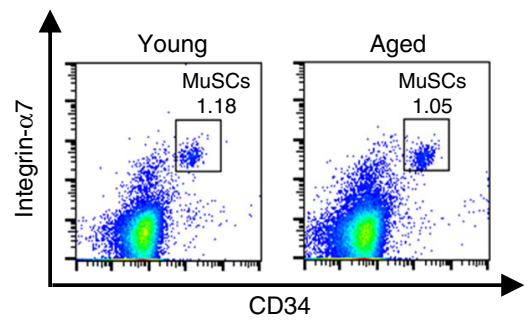

C

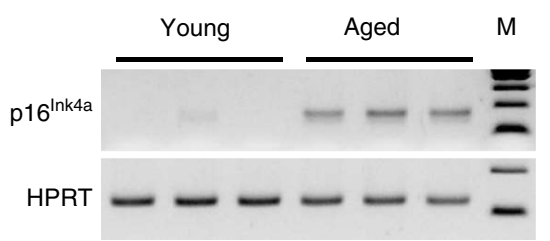

f

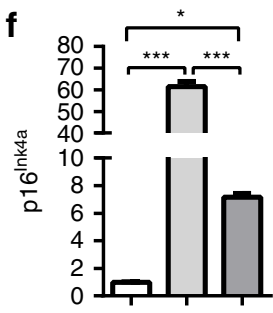

e
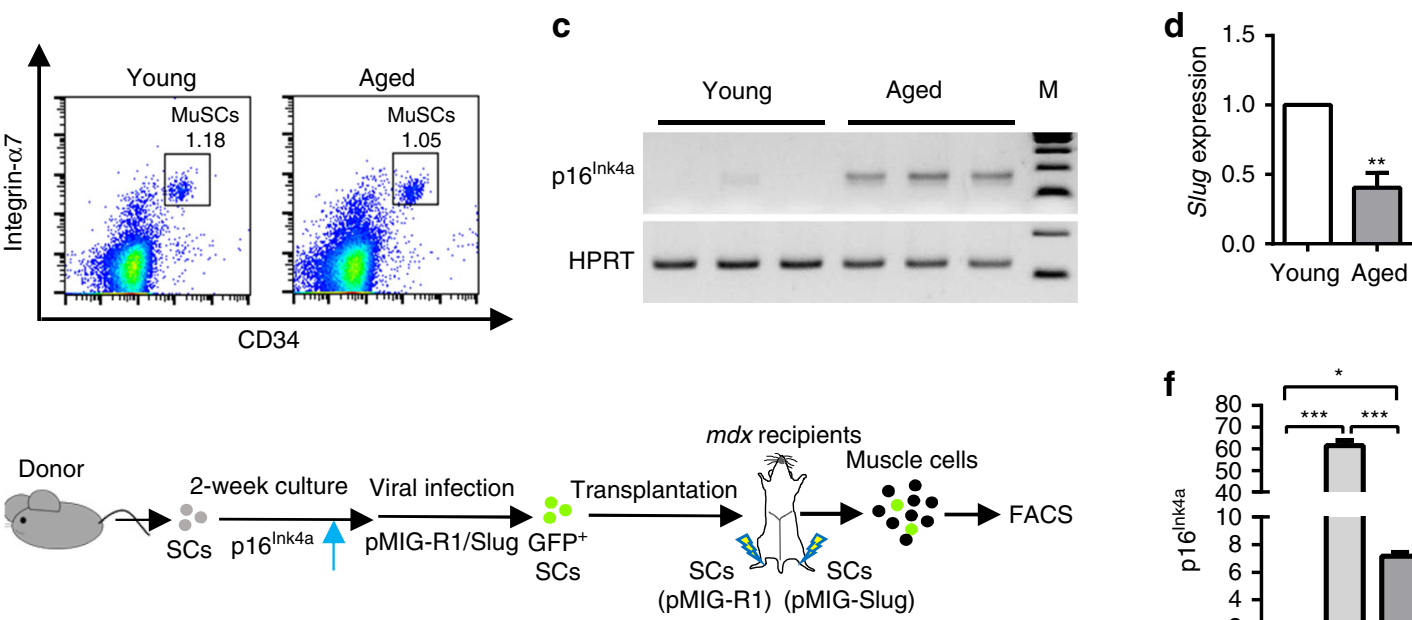
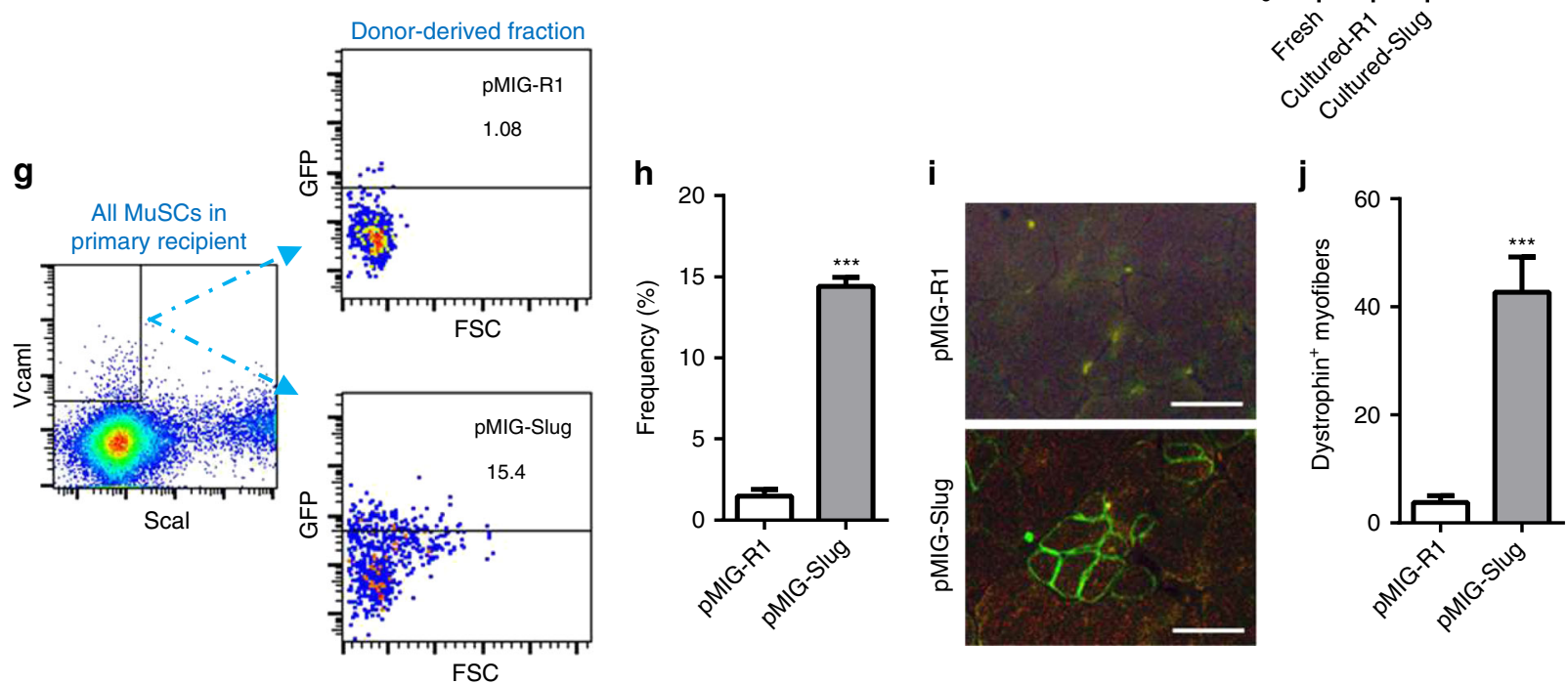

Taken as a whole, these findings demonstrated that lack of sufficient Slug expression is an important factor for derepression of p16 Ink4a in aged SCs, and that restoring Slug expression improves p16 ${ }^{\text {Ink4a }}{ }_{\text {-caused }}$ regenerative and self-renewing defects in SCs.

\section{Discussion}

Slug is a well-established regulatory transcriptional factor essential for epithelial-mesenchymal transition (EMT) during embryogenesis $^{34,35}$, tumor metastasis ${ }^{36,37}$, adult stem cells $^{38-41}$, and cellular reprogramming ${ }^{42,43}$. Slug is ubiquitously expressed in majority of normal tissues in the adult ${ }^{13}$. Slug knockout mice exhibit several severe abnormalities, including postnatal growth retardation ${ }^{44}$, attenuated acute cutaneous inflammatory response ${ }^{45}$, thinner epidermis ${ }^{46}$, and delayed hair follicle development ${ }^{47}$. These defects are typical phenotypes found in mice at advanced age, reminiscent of the essential role of Slug in aging. Here, we described an undiscovered function of Slug in MuSC aging. Our results illustrated that Slug is highly expressed in SCs, and acts as a transcriptional repressor of $p 16^{\text {Ink } 4 a}$. Lack of Slug 
Fig. 7 Slug Overexpression Restores Repopulating Capacity of Aged SCs. a Volcano plots demonstrating differentially expressed genes between freshly isolated young and aged SCs from the microarray data (GEO accession: GSE50821 and GSE72179) of indicated publications. Green dots indicate substantially increased while red dots indicated substantially decreased genes in aged SCs compared with young SCs. b Flow cytometric plotting of MuSCs (CD45-CD11b-CD31-Sca1-Integrin- $\alpha 7^{+}{ }^{+}{ }^{-}$D 34 $\left.{ }^{+}\right)$in young and aged mice. c Qualitative RT-PCR showing up-regulation of $p 16^{\text {Ink } 4 a}$ in aged SCs. HPRT was used as internal control for PCR. $\mathbf{d}$ qPCR analysis of relative mRNA levels of Slug in SCs from the young and aged mice $\left(n=3\right.$ mice per group). ${ }^{\star \star} p<0.01$ by student's $t$-test. e Scheme of SCs repopulation experiment. SCs were passaged weekly. After 2 weeks of culture, SC-derived myoblasts were infected with GFP-control (pMIGR1) or Slug-expressing (pMIG-Slug) retroviruses, and then transplanted into either side of pre-injured TA muscle of mdx recipient. Total mononucleated muscle cells were isolated separately from either TA muscle 4 weeks after transplantation, and subjected to flow cytometric analysis for the frequency of donor-derived SCs (GFP+). $\mathbf{f}$ Quantification of relative mRNA levels of $p 166^{\operatorname{lnk} 4 a}$ in quiescent and cultured primary mouse SCs with or without overexpressing Slug. ${ }^{\star} p<0.05,{ }^{\star \star \star} p<0.001$ by one-way ANOVA. $\mathbf{g}$ Representative flow cytometric analysis of the fraction of donor-derived SCs (pMIGR1 or pMIG-Slug) within the total recipient MuSC (CD45- CD31-Sca1 ${ }^{-}$Vcam I ${ }^{+}$) subpopulation in mononucleated TA muscle cells. $\mathbf{h}$ Percent of donor-derived cells $\left(\mathrm{GFP}^{+}\right)$in total recipient MuSC (CD45-CD31-Sca1- $1^{-}$cam I+) subpopulation of TA mononucleated muscle cells from mdx recipients $(n=3-4) .{ }^{* \star} p<0.001$ by student's $t$-test. i Representative dystrophin immunostaining (Scale bar, $50 \mu m$ ) on TA muscles transplanted with pMIGR1 or pMIGR1-Slug retrovirus-infected myoblasts. $\mathbf{j}$ Quantification of dystrophin-expressing myofibers in TA muscles of recipient $m d x$ mice $(n=6 \mathrm{mice}$ per group) shown in $\mathbf{i}{ }^{\star \star \star} p<0.001$, Student's $t$-test. Data are shown as mean \pm SEM of three independent replicates. Unprocessed gel blots are provided in the Supplementary Fig. 14 and source data file. Also see Supplementary Fig. 13. Source data are provided as a Source Data file

triggers SCs into pre-senescence state by accumulating p16 $^{\text {Ink4a }}$ expression and compromising SC self-renewing capacity after activation, leading to MuSC exhaustion and muscle regeneration defect. This study revealed a cell-autonomous mechanism of SCs losing their intrinsic regenerative and self-renewal capacities during chronological aging.

The role of Slug in skeletal muscle regeneration was firstly reported by the Hoffman group and they showed that Slug is a downstream target of MyoD and its expression was markedly increased at the whole muscle tissue level on day 4 and 10 during muscle regeneration ${ }^{33}$. Of note, they demonstrated that Slug knockout mice were defective for muscle regeneration after the first round of injury by cardiotoxin, which is different from our current findings in the global and conditional Slug knockout mouse models. Here, we demonstrated that Slug is highly expressed in quiescent SCs and its expression is greatly reduced in differentiated myotubes. This is consistent with the more recent work showing gradually diminished Slug protein in differentiating myoblasts in vitro ${ }^{22}$. Our current findings do not support that Slug is a downstream target of MyoD, since Slug expression is abundant in MyoD-negative quiescent SCs. As revealed by Soleimani et al., Snail/Slug compete with MyoD and prevent its occupancy on differentiation-specific regulatory elements in undifferentiated primary myoblasts ${ }^{22}$. Therefore, lack of competitive Slug binding should facilitate easier access of MyoD to those differentiation-regulating elements on its first appearance after injury and initiate myogenic program. Indeed, Slug-deficient mice were able to regenerate their muscle upon first round of muscle injury. In our current studies, we further proved that the failure of muscle repair in Slug-deficient mice after multiple injuries was partially due to the exhaustion of SC pool that was caused by their SC self-renewing defect. However, in view of the abundant expression of Slug in myoblasts, it is possible that Slug is playing a role outside of self-renewal, which could affect muscle regeneration and transplantation potential, by biasing SC proliferation versus differentiation.

In our current study, Slug deletion caused only a small increase of $p 16^{\text {Ink4a }}$ mRNA in quiescent SCs and did not prevent their activation and differentiation following the first muscle damage. We infer that the compensatory regulation of Snail, another member of the Snail/Slug superfamily of zinc-finger transcription factor, was probably also crucial to keep p16 ${ }^{\text {Ink4a }}$ transcription under control. This conjecture is supported by the fact that both Snail and Slug recognize and bind to the identical E-box motifs in myoblasts ${ }^{22}$. Interestingly, the Gridley group have reported a compensatory regulation of the Snail1 and Snail2 (Slug) during chondrogenesis ${ }^{48}$. Such compensation from Snail expression might also facilitate muscle regeneration as observed in Slugdeficient mice after first round of muscle injury as Snail can indirectly influence key myogenic transcription factors like Myf5 to activate myogenesis ${ }^{49}$. Of note, both Snail and Slug proteins decrease during myoblast differentiation ${ }^{22}$. Therefore, we reasoned that the large increase in $p 16^{\text {Ink } 4 a}$ mRNA expression in cultured Slug-deficient myoblasts and accumulated $\mathrm{p} 16^{\mathrm{Ink} 4 \mathrm{a}}$ protein in activated SCs were due to the loss of the dual regulation conferred by both Slug and Snail1. Future studies using Snai1 and Slug double knockout mice will be essential in testing this hypothesis. In addition, an increase in $p 16^{\text {Ink } 4 a}$ mRNA but not protein expression level in quiescent Slug ${ }^{-/}$SCs suggests a posttranslational regulation mechanism. It is possible that p16 $6^{\text {Ink } 4 a}$ protein is unstable in resting SCs. Indeed, it was reported that p16 ${ }^{\text {Ink } 4 a}$ translation is suppressed by miR-24 $4^{50}$, which is highly expressed in quiescent SCs but greatly downregulated in activated $\mathrm{SCs}^{51}$. These findings might explain our finding that $p 16^{\text {Ink4a }}$ mRNA but not protein accumulated in quiescent Slug ${ }^{-/-}$SCs.

Quiescent SCs rely on fatty acid and pyruvate oxidation to maintain a low metabolic rate in quiescence. The energy supply switches to glycolysis during early activation ${ }^{52}$, while both mitochondrial density and oxidative phosphorylation activity are increased following differentiation ${ }^{53-55}$. It was previously demonstrated that the mitochondrial-associated metabolism pathway is more silent in Pax $7^{\mathrm{Hi}} \mathrm{SCs}$ being of higher level of stemness and responsible for self-renewal ${ }^{56}$. A more recent study of in vitro culture of SCs in conditioned medium favoring either glycolysis or oxidative phosphorylation showed that a shift from glycolysis to oxidative phosphorylation negatively affects the return to quiescence of activated SCs ${ }^{57}$. Although SCs remain in quiescence in the absence of Slug, a batch of genes in glycolysis, oxidative phosphorylation, and nutrient-sensitive PI3K-AKTmTOR pathways were upregulated therein. Such metabolic gene expression profiling is consistent with our observation of the attenuated self-renewal and accelerated senescence in Slug null SCs.

An aging-associated reduction of Slug/Snai2 expression was observed in mouse SCs. Mechanistically, Slug expression was reported to be under control of a number of signaling pathways such as FGF, Wnt, TGF $\beta$, Notch, stem cell factor (SCF), integrins, and estrogens etc. ${ }^{58}$. Several of these signaling molecules including FGF $^{3}$ and Notch ${ }^{59,60}$ are known as inducers of Slug and were reported to decline with age in mouse SCs. Therefore, we speculate that these impaired upstream signaling pathways might account for Slug insufficiency in aged SCs. Interestingly, active Notch and Notch ligand Delta are also decreased in old human muscle when compared to young 
muscle ${ }^{61}$. Indeed, we identified the potential Slug-binding consensus sequence (E-box) in the promoter region of human p16 ${ }^{\text {Ink } 4 a}$ gene as well (Supplementary Fig. 9c). Gain and loss of function of SLUG in primary human myoblasts causes down and upregulation of $p 16^{I N K 4 A}$ expression, respectively (Supplementary Fig. 9d-g). Function of Slug in SCs might therefore be highly conserved in mouse and human. Future studies exploring small molecules that are able to induce Slug expression in aged mice are warranted to test the improvement of aging-associated muscle stem cell defects in vivo.

In conclusion, although $\mathrm{p} 16^{\text {Ink4a }}$ has been well recognized as a key factor for stem cell aging, upstream regulators for causing its dysregulation remain unknown. Our studies demonstrated that Slug plays an important role in repressing $p 16^{\text {Ink } 4 a}$ transcription in skeletal MuSCs. Loss of Slug gives rise to severe regenerative defects during continuous muscle injury by accelerating entry of SC senescence. Restoration of Slug expression during chronological aging rejuvenates the function of aged SCs by suppressing $p 16^{\operatorname{Ink} 4 a}$ expression. These results offer a promising therapeutic target for aging-associated degenerative muscle disease.

\begin{abstract}
Methods
Mice. C57BL/6, C57BL/6-Tg(CAG-EGFP)10sb/J, Pax $7^{t m 1(c r e) M r c / J, ~ B 6 . C g-P a x ~} 7^{t m 1}$ ${ }^{(c r e / E R T 2)} \mathrm{Gaka} / \mathrm{J}$, and C57BL/10ScSn-Dmd ${ }^{m d_{x}} / \mathrm{J}$ mice were purchased from Jackson Laboratories. $16^{\text {Ink4a }}$ knockout mice were obtained from the NCI Mouse Repository. Pax7-zsGreen transgenic mice were a kind gift from Dr. M. Kyba (University of Minnesota). Slug conditional knockout mouse line was generated in the current study. All compound genetically-engineered mice were a result of breeding the above strains followed by appropriate PCR-based genotyping. From 4 to 8-week-old male mice were used as adult mice in all the experiments, while 2year-old male mice were used as aged mice. All the animal studies were approved by the Animal Care and Use Committee (approved protocol number: 16-111) and performed in compliance with the institutional guidelines of the University of Illinois at Chicago.
\end{abstract}

Gene targeting and generation of a Slug-floxed allele. The Slug targeting construct was generated by PCR using 129X1/svJ mouse genomic DNA. Primers for generating the targeting construct are shown in Supplementary Table 1. First, a $\sim 3 \mathrm{~Kb}$ genomic sequence containing Slug promoter region was PCR-amplified as the left arm with SlugKO-P1 and SlugKO-P2 primers and cloned into NotI and SalI sites of pLoxP-2FRT vector (designated as pLoxP-2FRT_SlugN). Second, $\mathrm{a} \sim 4 \mathrm{~Kb}$ genomic sequence covering the genomic region from the immediate downstream of SlugKO-P2 binding site to the intron 3 was PCR-amplified as the right arm with SlugKO-P3 and SlugKO-P4 primers, followed by cloning into $\mathrm{BamH1}$ and Kpnl sites of pBS vector (designated as pBS_SlugC). Third, a LoxP linker was cloned into EcoR1 site of pBS_SlugC and the resultant plasmid was named as pBS_SlugC-LoxP. Lastly, the SlugC-LoxP fragment was isolated from pBS_SlugC-LoxP with BamH1 and Kpn1 and then cloned into pLoxP-

2FRT_SlugN. The resulting construct was designated as p2LoxP-2FRT_Slug, which carries neomycin (neo) for positive selection and HSV-tk marker for negative selection. The linearized targeting construct was nucleofected into R1 ES cells (129/svx129/Sv-CP F1) by Nucleofector II (Lonza, Allendale, NJ), and followed by positive selection with G418 and negative selection with FIAU. Correctly targeted ES cells were first screened for the presence of the DNA sequence containing LoxP and FRT sites by PCR with SlugKO-P11 and Neo-R1 primers, then screened for the left arm by long-PCR with SlugKO-P10 and Neo-R1 and confirmed by DNA sequencing. Two positive ES cell clones were expanded and microinjected into C57BL/6 blastocysts to generate chimeric Slugflox-neo/+ founder mice at the Mouse Transgenic \& Gene Targeting Core of Maine Medical Center Research Institute. The neo cassette was deleted from the germline by crossing Slugflox-neo/+ mice to Flp-expressing transgenic mice (ACTB:FLPe B6; SJL, The Jackson Laboratory), which express Flp recombinase under the control of the human actin promoter ${ }^{62}$. The Flp gene was subsequently removed by backcrossing with C57BL/6 mice to generate $\operatorname{Slug} f l /+$ mice. The Slugfl/+ allele genotyping was performed by PCR with SlugPT-F and SlugEX1-R primers (Supplementary Table 1).

Generation of SC-specific Slug knockout Mouse Line. Female Slugfl/fl mice were bred with male mice $\left(\operatorname{Pax} 7^{t m 1}(\mathrm{cre}) M r c / \mathrm{J}\right)$ homozygous for the Cre-recombinase gene under the control of endogenous Pax7-promoter to generate SC-specific Slug knockout mouse line (Slugfl/fl Pax $7^{C r e /+}$ or Slugfl/fl Pax $\left.7^{C r e E R}\right)$. Genotyping for Cre gene was performed using a multiplex PCR method according to the provided protocol. To delete Slug in adult SCs, tamoxifen (Sigma) was administered intraperitoneally ( $100 \mathrm{mg} \mathrm{kg}^{-1}$ body weight per day) for 5 consecutive days.
Muscle stem cell isolation. Murine hindlimb skeletal muscles were dissected, cleaned by removal of non-muscle tissues and subjected to a gentle collagenase (100 U ml ${ }^{-1}$, Catalog no. 171001015, Life technologies, Carlsbad) and dispase (2 $\mathrm{U} \mathrm{ml}^{-1}$, Catalog no. 17105041, Life technologies, Carlsbad) digestion in DMEM at $37^{\circ} \mathrm{C}$ water bath for $1 \mathrm{~h}$. Enzymatically digested muscle mass was re-suspended in DMEM with $10 \%$ horse serum, and further mechanically triturated through vigorous and consecutive passing through a $10-\mathrm{ml}$ glass pipette and subsequently 9" cotton-plugged glass Pasteur pipette. Resultant cell suspension was passed through sequential $70-\mu \mathrm{m}$ and $40-\mu \mathrm{m}$ strainer (BD Bioscience, San Jose, USA) to generate bulk skeletal muscle single-cell suspensions.

To isolate MuSCs from undamaged muscles, mononucleated cells were stained with biotinylated antibodies reactive to $\mathrm{CD} 45, \mathrm{CD} 11 \mathrm{~b}, \mathrm{CD} 31$, and Sca1, and then incubated with streptavidin-APC-Cy7, integrin- $\alpha 7$ antibody labeled with $\mathrm{PE}$ (MBL, Woburn, USA), and eFluor660-conjugated CD34 antibody (eBioscience, San Diego, USA). Cells negative for CD45, CD31, CD11b, and Sca1 but positive for CD34 and integrin- $\alpha 7$ were sorted as MuSCs by fluorescence-activated cell sorting (FACS) ${ }^{63}$.

To isolate MuSCs from damaged muscles, mononucleated cells were first stained with biotinylated antibodies reactive to CD31 and CD45, followed by incubation with streptavidin-APC-Cy7, Scal antibody labeled with FITC (eBioscience, San Diego, USA), and PE-conjugated Vcam I antibody (Miltenyl Biotec, Bergisch Gladbach, Germany). Cells negative for CD31, CD45, and Sca1 but positive for Vcam I were sorted as MuSCs by FACS ${ }^{19}$.

To isolated MuSCs from Pax7-zsGreen transgenic mice, $z^{2}$ Green ${ }^{+}$cells were directly sorted from mononucleated cells by FACS.

Myofiber culture and AraC treatment. EDL muscles were dissected and digested for $1 \mathrm{~h}$ in DMEM with $0.2 \%$ Collagenase type I. Pre-digested EDLs were then transferred to horse serum-precoated dish containing prewarmed DMEM, and gently flushed with a fire polished large bore glass pipette to release myofibers. Live and healthy single myofibers were obtained by three consecutive transfers of individual myofibers with small bore glass pipette into new horse serum-precoated dishes containing prewarmed DMEM. Live myofibers were cultured in uncoated plastic 24-well-plates at density of 1 fiber per well with DMEM supplemented with $15 \%$ horse serum and $1 \mathrm{nM}$ bFGF (PROSPEC, East Brunswick, USA). For AraC experiment, myofibers were cultured for $72 \mathrm{~h}$ and then incubated with or without $100-\mu \mathrm{M}$ AraC (C1768, Sigma, USA) for $48 \mathrm{~h}$ (from day 3-5) and fixed (day 5) for immunostaining.

Skeletal muscle cell culture and differentiation. Mouse skeletal MuSCs were cultured using the MuSC growth medium [DMEM/F10 (1:1) with 20\% FBS, $2.5 \mathrm{ng}$ $\mathrm{ml}^{-1}$ bFGF (PROSPEC, East Brunswick, USA) and 1\% penicillin/streptomycin] in Matrigel (BD Biosciences, San Jose, USA) precoated tissue culture plates. To induce differentiation, cells were switched from growth medium into the fusion medium (DMEM supplemented with $5 \%$ horse serum) for 5 days.

Comparison of proliferation potential. Cumulative population doubling level (CPDL) was calculated using the formula $\chi=\left[\log _{10}\left(N_{H}\right)-\log _{10}\left(N_{1}\right)\right] / \log _{10}(2)^{64}$ in which $N_{1}$ is the inoculum cell number and $N_{H}$ is the cell harvest number. To determine cumulated doubling level, population doubling level for each passage was calculated and then added to the levels of the previous passages. Cumulative doubling number was first calculated from passage 1 .

Histology and immunohistochemistry in muscle cryosections. Fresh TA mus cles were embedded in Tissue-Tek ${ }^{\circledast}$ O.C.T ${ }^{\mathrm{TM}}$ compound (Fisher Scientific, Hampton, USA), frozen in liquid nitrogen-cooled isopentane, and stored at $-80^{\circ} \mathrm{C}$ until analysis. Frozen muscles were cross-sectioned $(10 \mu \mathrm{m})$ using a Leica CM1850 cryostat. For histology, the sections were dried at room temperature for over $3 \mathrm{~h}$ before staining. Sections were then rehydrated in PBS for $5 \mathrm{~min}$ and fixed in $10 \%$ formalin for $15 \mathrm{~min}$, and proceeded with routine haematoxylin and eoxin (H\&E) staining. For immunohistochemistry (IHC) study, air-dried muscle sections were fixed with $4 \%$ paraformaldehyde (PFA) and permeabilized in $0.25 \%$ Trition X-100. Tissue sections were then blocked in phosphate buffer saline (PBS) with 5\% goat serum, $2 \%$ bovine serum albumin, and $1 \%$ Tween- 20 for $1 \mathrm{~h}$, and then followed by incubation with anti-Pax7 (DSHB, Iowa, USA, 1:10), anti-Laminin (clone A5, Invitrogen, Carlsbad, USA, 1:100 dilution), anti-p16 (M-156, Santa Cruz Biotechnology, USA), anti-Ki67 (Clone SolA15, eBioscience, USA, 1:100), and/or antidystrophin (Clone MANDYS8, Sigma, USA, 1:100) primary antibodies overnight at $4{ }^{\circ} \mathrm{C}$. After washing with PBS, sections were then incubated with Alexa-conjugated secondary antibodies (Invitrogen, Carlsbad, USA, 1:200 dilution).

Immunofluorescence staining. Freshly isolated SCs were cytospun onto slides and fixed with $4 \%$ PFA. After being permeabilized by $0.2 \%$ Trition X-100, cells were then blocked in PBS with $1 \%$ bovine serum albumin (BSA) for $1 \mathrm{~h}$ at room temperature followed by staining with anti-Pax7 (DSHB, Iowa, USA, 1:10) and anti-Slug (Clone C19G7, Cell Signaling, USA, 1:400) primary antibodies overnight at $4{ }^{\circ} \mathrm{C}$. Cells were washed three times with PBS and incubated with appropriate secondary antibody for $1 \mathrm{~h}$ at room temperature. 
SA- $\boldsymbol{\beta}$-galactosidase staining. Cellular senescence was evaluated by $\beta$ galactosidase activity using MarkerGene ${ }^{\mathrm{TM}}$ Cellular Senescence Assay Kit (Marker Gene, Eugene OR, USA). Briefly, cells or tissue sections were fixed for 4 and 20 min, respectively. Fixed samples were then washed in PBS (pH7.0) twice for $10 \mathrm{~min}$ and incubated with $\mathrm{X}$-gal containing staining buffer at $37^{\circ} \mathrm{C}$ overnight for cells and $48 \mathrm{~h}$ for tissue sections. For tissue sections, X-gal substrate was changed after $24 \mathrm{~h}$. Samples were washed in PBS, and post-fixed in 1\% PFA $5 \mathrm{~min}$ for cells and $30 \mathrm{~min}$ for sections. After being washed three times for $10 \mathrm{~min}$ in PBS, samples were mounted in PBS, $20 \%$ glycerol or proceeded for IHC.

Muscle injury and SC transplantation. Experimental mice were anesthetized by intraperitoneal injection of ketamine $\left(100 \mathrm{mg} \mathrm{kg}^{-1}\right.$ body weight) and xylazine (10 $\mathrm{mg} \mathrm{kg}^{-1}$ body weight). For single muscle injury, TA muscles were injected with $50 \mu \mathrm{l}$ of $1.2 \% \mathrm{BaCl}_{2}$ (B0750, Sigma, USA) at multiple sites and harvested 10 days post injury. For double or triple injuries, mice were allowed to recover for 1 month after last $\mathrm{BaCl}_{2}$ injection, and then injured again with $50 \mu \mathrm{l}$ of $1.2 \% \mathrm{BaCl}_{2}$. For in vivo cellular senescence detection, TA muscles were injured with $50 \mu \mathrm{l}$ cardiotoxin (Naja pallida, $10 \mu \mathrm{M}$; Calbiochem, USA). For SC transplantation, recipient $m d x$ mice were anesthetized by intraperitoneal injection of ketamine $\left(100 \mathrm{mg} \mathrm{kg}^{-1}\right.$ body weight) and xylazine (10 $\mathrm{mg} \mathrm{kg}^{-1}$ body weight). TA muscles were injected with $50 \mu \mathrm{l}$ of $1.2 \% \mathrm{BaCl}_{2} 1$ day before transplantation. Next day, SCs (or myoblasts) were re-suspended in $15 \mu \mathrm{l}$ of PBS and then injected into pre-injured TA muscle. TA muscles were harvested from recipient mice 4 weeks after transplantation and analyzed by cryosectioning and microscopy.

RNA extraction and qPCR. Total RNA was extracted from samples (SCs or myoblasts) using Quick-RNA ${ }^{\mathrm{TM}}$ MicroPrep kit (ZYMO Research, USA). First strand cDNA synthesis was performed using $\mathrm{RT}^{\mathrm{TM}}$ Master Mix (LAMDA BIOTECH). RT-PCR was performed using GoTaq ${ }^{\oplus}$ Green Master Mix (Promega, Madison, USA) on Bio-Rad Thermal Cycler. qPCR was performed using Bullseye EvaGreen Qpcr MasterMix (MIDSCI, USA). The primer sequences for PCR are listed in Supplementary Table 1.

cDNA microarray and data analysis. Total RNAs extracted using Quick RNA ${ }^{\mathrm{TM}}$ MicroPrep kit (ZYMO Research, Irvine, USA) were first analyzed by Bioanalyzer (Agilent Technologies, Santa, Clara, USA). A triplicate of RNA samples with an RNA integrity number (RIN) $>9.0$ were used for subsequent labeling and hybridization with Mouse Gene 2.0 ST Arrays (Affymetrix, USA). Expression data were processed using Gene Expression Console software (Affymetrix, Santa Clara, USA). The significance of differentially expressed genes was determined using Transcriptome Analysis Console software (Affymetrix, Santa Clara, USA). GSEA version 3.0 (http://software.broadinstitute.org/gsea) and MSigDB gene sets version 6.1 were used to determine the sets of genes that are statistically different between two groups using GSEA preranked mode ${ }^{25}$. Enrichment Map ${ }^{65,66}$ was used to visualize enriched pathways. GSEA output files were subjected to the Enrichment Map app with the following cutoff parameters to build the map: $\mathrm{p}$ value $<0.001$, FDR qvalue $<0.05$ and overlap similarity coefficient $>0.5$. Nodes in the Enrichment Map represent a set of genes and their connections in which the set of genes have in common nodes. The edge thickness is proportional to the overlap of two gene sets. The node colors map enrichment significance: blue/downregulated, red/upregulated. Gene ontology analysis (GO) on retrieved RNA-seq dataset (GSM937218) was conducted in the Database for Annotation, Visualization, and Integrated Discovery v6.7 (DAVID, http://david.abcc.ncifcrf.gov) using all detectable genes as the background. Only enriched pathways with FDR value $<0.05$ are presented.

Retroviral transduction of myogenic progenitor cells. Two hundred ninetythree T cells were transfected with a mixture of DNA plasmids containing $1.5 \mu \mathrm{g}$ of Pcl-Eco (IMGENEX), $2.5 \mu \mathrm{g}$ of pMIGR1 vector or pMIGR1 harboring Slug by Fugene HD (Roche) according to the manufacturer's instruction. Virus-containing media were collected $24 \mathrm{~h}$ after transfection and filtered through a $0.45-\mu \mathrm{m}$ poresize filter. SCs were transduced with retrovirus-containing media supplemented with $4-\mu \mathrm{g} \mathrm{ml}^{-1}$ polybrene (Sigma), followed by centrifugation at $1000 \times g$ for $1 \mathrm{~h}$.

ChIP assay. ChIP assay was performed with ChIP-IT Express Enzymatic kit (Active Motif, Carlsbad, CA, USA) according to the manufacturer's instruction. Genomic DNA was pulled down by anti-Flag (FG4R, Thermo Scientific, USA), anti-HA (Thermo Scientific, USA), or anti-IgG (Millipore, USA) control antibodies. Primers for amplifying promoter region of $\mathrm{p} 16^{\text {Ink4a }}$ from immunoprecipitated DNA fragments are listed in Supplementary Table 1

Tamoxifen induced SC-conditional Slug knockout. Experimental mice were intraperitoneally injected with tamoxifen $\left(100 \mathrm{mg} \mathrm{kg}^{-1}\right.$ per day) for 5 days. First $\mathrm{BaCl}_{2}$ injury was conducted in TA muscles 5 days after the last injection of tamoxifen. For double injury, mice were recovered for 1 month after the first injury, and subject to a second $\mathrm{BaCl}_{2}$ injection at the same sites. TA muscles were harvested on day 10 post each injury.
Epitope Flag/HATagging by CRISPR/Cas9-based genome editing. A gRNA targeting a preselected site near the stop code (TGA) in the exon 3 of Slug gene was designed (left panel) and cloned into pAAV-gRNA-GFP expression vector. The donor template plasmid contains a DNA sequence encoding three copies of HA and Flag tags and blasticidin resistance gene (Blast), which is flanked by the twohomology left and a right arm sequence. The blasticidin sequence is in-frame fused to the FLAG tag via a P2A sequence. The three copies of HA tag is in-frame fused to the end of Slug (right before stop code). The PAM sequence (TGG) was changed to "TaG" in the left arm of the donor vector to avoid binding by the gRNA, but without altering protein sequence (right panel). Myoblasts were co-transfected with Cas9 and/or the gRNA expression vectors, together with the donor template plasmid. Three days later, blasticidin was added into culture medium to selected cells with correct site-directed integration of the donor sequence (three copies of HA and FLAG tags and Blast).

Luciferase reporter assay. Myoblast cells were seed in 48 -well plates and cultured in myoblast growth medium. A total of $0.5 \mu \mathrm{g}$ of DNA containing $0.1 \mu \mathrm{g}$ of PGl3-p16 luciferase reporter plasmid, $0.4 \mu \mathrm{g}$ of expression plasmid (pMIG-R1 or pMIG-Slug) was transfected using PEI. A co-transfected $0.025 \mu$ G CMV-lacZ plasmid was used to normalize transfection efficiency. Cells were lysed $24 \mathrm{~h}$ using Glo Lysis Buffer (E266A, Promega) after transfection, and the luciferase activity of each extract was assayed using Bright-Glo ${ }^{\mathrm{TM}}$ Luciferase Assay System (E2620, Promega). One microliter cell lysate was added into $399-\mu \mathrm{l} Z$ buffer. One hundred microliter ONPG (o-nitrophenyl b-D-galactopyranoside; Sigma \#N1127) with concentration of $4 \mathrm{mg} / \mathrm{ml}$ was added and mixed well. When the sample become yellow, $500 \mu \mathrm{l} 1 \mathrm{M}$ carbonate was added to stop the reaction and the activity of beta-galactosidase was measured at A420 to normalize the luciferase activity.

Western blot analysis. Total protein was lysed using CelLytic ${ }^{\mathrm{TM}}$ MT Cell Lysis reagent (\#C3228, Sigma, USA) supplemented with protease inhibitor cocktail (\#P8340, Sigma, USA). Cell lysates were centrifuged for $15 \mathrm{~min}$ at $13,000 \times g$, and supernatants were collected for protein concentration determination using Pier$\mathrm{ce}^{\mathrm{TM}}$ BCA Protein Assay kit (\#23225, ThermoFisher Scientific). Fifteen micrograms of total protein from each sample were resolved by $10 \%$ SDS-PAGE and electroblotted onto polyvinylidene difluoride membranes. Immunoblotting was performed with anti-Flag (FG4R, Thermo Scientific, USA), anti-HA (5B1D10, Thermo Scientific, USA), anti- $\beta$-actin (Cell Signaling Technology \#4970, USA), and anti-Slug (\#9585, Cell Signaling Technology, USA) antibodies at $4{ }^{\circ} \mathrm{C}$ overnight. After washing, membranes were incubated with a horseradish peroxidaseconjugated goat anti-rabbit IgG antibody (\#1705046, Bio-Rad, USA) for $1 \mathrm{~h}$ at room temperature. Bound antibodies were detected using Clarity Max ${ }^{\mathrm{TM}}$ Western ECL substrate (\#68SC, Bio-Rad, USA). Uncropped blots can be found in Supplementary Fig 14 and the Source data file.

\section{Data availability}

Data supporting the findings of this manuscript are available within the article and its supplementary information files or from the corresponding author upon reasonable request.

All data from DNA microarray experiments have been deposited in Gene Expression Omnibus database under accession code GSE128507.

The source data underlying Fig. 1-7 and Supplementary Figs 1, 3-5, 8-10 and 12 are provided as a Source Data file.

Received: 17 January 2018 Accepted: 14 May 2019

Published online: 12 June 2019

\section{References}

1. Frontera, W. R., Hughes, V. A., Lutz, K. J. \& Evans, W. J. A cross-sectional study of muscle strength and mass in 45- to 78-yr-old men and women. J. Appl. Physiol. (1985) 71, 644-650 (1991).

2. Carlson, B. M. \& Faulkner, J. A. Muscle transplantation between young and old rats: age of host determines recovery. Am. J. Physiol. 256, C1262-C1266 (1989)

3. Chakkalakal, J. V., Jones, K. M., Basson, M. A. \& Brack, A. S. The aged niche disrupts muscle stem cell quiescence. Nature 490, 355-360 (2012).

4. Sinha, M. et al. Restoring systemic GDF11 levels reverses age-related dysfunction in mouse skeletal muscle. Science 344, 649-652 (2014).

5. Sousa-Victor, P. et al. Geriatric muscle stem cells switch reversible quiescence into senescence. Nature 506, 316-321 (2014).

6. Bernet, J. D. et al. p38 MAPK signaling underlies a cell-autonomous loss of stem cell self-renewal in skeletal muscle of aged mice. Nat. Med. 20, 265-271 (2014). 
7. Cosgrove, B. D. et al. Rejuvenation of the muscle stem cell population restores strength to injured aged muscles. Nat. Med. 20, 255-264 (2014).

8. Montarras, D. et al. Direct isolation of satellite cells for skeletal muscle regeneration. Science 309, 2064-2067 (2005).

9. Relaix, F. \& Zammit, P. S. Satellite cells are essential for skeletal muscle regeneration: the cell on the edge returns centre stage. Development 139, 2845-2856 (2012).

10. Lopez-Otin, C., Blasco, M. A., Partridge, L., Serrano, M. \& Kroemer, G. The hallmarks of aging. Cell 153, 1194-1217 (2013).

11. Sharpless, N. E. \& Sherr, C. J. Forging a signature of in vivo senescence. Nat. Rev. Cancer 15, 397-408 (2015).

12. Sherr, C. J. Ink4-Arf locus in cancer and aging. Wiley Inter. Rev. Dev. Biol. 1, 731-741 (2012).

13. Parent, A. E., Choi, C., Caudy, K., Gridley, T. \& Kusewitt, D. F. The developmental transcription factor slug is widely expressed in tissues of adult mice. J. Histochem. Cytochem. 52, 959-965 (2004).

14. Pallafacchina, G., Blaauw, B. \& Schiaffino, S. Role of satellite cells in muscle growth and maintenance of muscle mass. Nutr. Metab. Cardiovasc. Dis. 23 (Suppl 1), S12-S18 (2013).

15. Yin, H., Price, F. \& Rudnicki, M. A. Satellite cells and the muscle stem cell niche. Physiol. Rev. 93, 23-67 (2013).

16. Shefer, G., Van de Mark, D. P., Richardson, J. B. \& Yablonka-Reuveni, Z. Satellite-cell pool size does matter: defining the myogenic potency of aging skeletal muscle. Dev. Biol. 294, 50-66 (2006).

17. Arpke, R. W. \& Kyba, M. . Flowcytometry and transplantation-based quantitative assays for satellite cell self-renewal and differentiation. Methods Mol. Biol. 1460, 163-179 (2016).

18. Hardy, D. et al. Comparative study of injury models for studying muscle regeneration in mice. PLOS ONE 11, e0147198 (2016).

19. Liu, L., Cheung, T. H., Charville, G. W. \& Rando, T. A. Isolation of skeletal muscle stem cells by fluorescence-activated cell sorting. Nat. Protoc. 10, 1612-1624 (2015).

20. Harrison, D. E. Competitive repopulation: a new assay for long-term stem cell functional capacity. Blood 55, 77-81 (1980).

21. Troy, A. et al. Coordination of satellite cell activation and self-renewal by Parcomplex-dependent asymmetric activation of p38alpha/beta MAPK. Cell Stem Cell 11, 541-553 (2012).

22. Soleimani, V. D. et al. Snail regulates MyoD binding-site occupancy to direct enhancer switching and differentiation-specific transcription in myogenesis. Mol. Cell 47, 457-468 (2012).

23. Janzen, V. et al. Stem-cell ageing modified by the cyclin-dependent kinase inhibitor p16INK4a. Nature 443, 421-426 (2006).

24. Boquoi, A. et al. Reversible cell cycle inhibition and premature aging features imposed by conditional expression of p16Ink4a. Aging Cell 14, 139-147 (2015).

25. Subramanian, A. et al. Gene set enrichment analysis: a knowledge-based approach for interpreting genome-wide expression profiles. Proc. Natl Acad. Sci. USA 102, 15545-15550 (2005).

26. Kataoka, H. et al. A novel snail-related transcription factor Smuc regulates basic helix-loop-helix transcription factor activities via specific E-box motifs. Nucleic Acids Res 28, 626-633 (2000).

27. Nieto, M. A. The snail superfamily of zinc-finger transcription factors. Nat. Rev. Mol. Cell Biol. 3, 155-166 (2002).

28. Wang, W., Martindale, J. L., Yang, X., Chrest, F. J. \& Gorospe, M. Increased stability of the p16 mRNA with replicative senescence. EMBO Rep. 6, 158-164 (2005).

29. Yoshida, N., Yoshida, S., Koishi, K., Masuda, K. \& Nabeshima, Y. Cell heterogeneity upon myogenic differentiation: down-regulation of MyoD and Myf-5 generates 'reserve cells'. J. Cell Sci. 111(Pt 6), 769-779 (1998).

30. Garcia-Prat, L. et al. Autophagy maintains stemness by preventing senescence. Nature 529, 37-42 (2016).

31. Oh, J. et al. Age-associated NF-kappaB signaling in myofibers alters the satellite cell niche and re-strains muscle stem cell function. Aging (Albany NY) 8, 2871-2896 (2016)

32. Bigot, A. et al. Replicative aging down-regulates the myogenic regulatory factors in human myoblasts. Biol. Cell 100, 189-199 (2008).

33. Zhu, C. H. et al. Cellular senescence in human myoblasts is overcome by human telomerase reverse transcriptase and cyclin-dependent kinase 4: consequences in aging muscle and therapeutic strategies for muscular dystrophies. Aging Cell 6, 515-523 (2007).

34. Savagner, P., Yamada, K. M. \& Thiery, J. P. The zinc-finger protein slug causes desmosome dissociation, an initial and necessary step for growth factorinduced epithelial-mesenchymal transition. J. Cell Biol. 137, 1403-1419 (1997).

35. Savagner, P. et al. Developmental transcription factor slug is required for effective re-epithelialization by adult keratinocytes. J. Cell Physiol. 202, 858-866 (2005).
36. Alves, C. C., Carneiro, F., Hoefler, H. \& Becker, K. F. Role of the epithelialmesenchymal transition regulator Slug in primary human cancers. Front Biosci. (Landmark Ed.) 14, 3035-3050 (2009).

37. Shih, J. Y. \& Yang, P. C. The EMT regulator slug and lung carcinogenesis. Carcinogenesis 32, 1299-1304 (2011).

38. Wu, W. S. et al. Slug antagonizes p53-mediated apoptosis of hematopoietic progenitors by repressing puma. Cell 123, 641-653 (2005)

39. Sun, Y., Shao, L., Bai, H., Wang, Z. Z. \& Wu, W. S. Slug deficiency enhances self-renewal of hematopoietic stem cells during hematopoietic regeneration. Blood 115, 1709-1717 (2010).

40. Tang, Y., Feinberg, T., Keller, E. T., Li, X. Y. \& Weiss, S. J. Snail/Slug binding interactions with YAP/TAZ control skeletal stem cell self-renewal and differentiation. Nat. Cell Biol. 18, 917-929 (2016).

41. Guo, W. et al. Slug and Sox9 cooperatively determine the mammary stem cell state. Cell 148, 1015-1028 (2012)

42. Liu, X. et al. Sequential introduction of reprogramming factors reveals a time-sensitive requirement for individual factors and a sequential EMTMET mechanism for optimal reprogramming. Nat. Cell Biol. 15, 829-838 (2013).

43. Gingold, J. A. et al. A genome-wide RNAi screen identifies opposing functions of Snail and Snai2 on the Nanog dependency in reprogramming. Mol. Cell 56, 140-152 (2014).

44. Jiang, R., Lan, Y., Norton, C. R., Sundberg, J. P. \& Gridley, T. The Slug gene is not essential for mesoderm or neural crest development in mice. Dev. Biol. 198, 277-285 (1998).

45. Newkirk, K. M. et al. The acute cutaneous inflammatory response is attenuated in Slug-knockout mice. Lab Invest 88, 831-841 (2008)

46. Newkirk, K. M. et al. Snai2 expression enhances ultraviolet radiation-induced skin carcinogenesis. Am. J. Pathol. 171, 1629-1639 (2007).

47. Parent, A. E., Newkirk, K. M. \& Kusewitt, D. F. Slug (Snai2) expression during skin and hair follicle development. J. Invest Dermatol 130, 1737-1739 (2010).

48. Chen, Y. \& Gridley, T. Compensatory regulation of the Snail and Snai2 genes during chondrogenesis. J. Bone Min. Res 28, 1412-1421 (2013).

49. Sieiro D., Rios A. C., Hirst C. E., Marcelle C. Cytoplasmic NOTCH and membrane-derived beta-catenin link cell fate choice to epithelialmesenchymal transition during myogenesis. Elife 5, e14847 (2016).

50. Lal, A. et al. p16(INK4a) translation suppressed by miR-24. PLoS ONE 3, e1864 (2008).

51. Sun, Y. et al. miR-24 and miR-122 negatively regulate the transforming growth factor-beta/smad signaling pathway in skeletal muscle fibrosis. Mol. Ther. Nucleic Acids 11, 528-537 (2018).

52. Cerletti, M., Jang, Y. C., Finley, L. W., Haigis, M. C. \& Wagers, A. J. Short-term calorie restriction enhances skeletal muscle stem cell function. Cell Stem Cell 10, 515-519 (2012).

53. Leary, S. C., Battersby, B. J., Hansford, R. G. \& Moyes, C. D. Interactions between bioenergetics and mitochondrial biogenesis. Biochim Biophys. Acta 1365, 522-530 (1998)

54. Lyons, C. N., Leary, S. C. \& Moyes, C. D. Bioenergetic remodeling during cellular differentiation: changes in cytochrome $c$ oxidase regulation do not affect the metabolic phenotype. Biochem. Cell Biol. 82, 391-399 (2004).

55. Kraft, C. S. et al. Control of mitochondrial biogenesis during myogenesis. Am. J. Physiol. Cell Physiol. 290, C1119-C1127 (2006).

56. Rocheteau, P., Gayraud-Morel, B., Siegl-Cachedenier, I., Blasco, M. A. \& Tajbakhsh, S. A subpopulation of adult skeletal muscle stem cells retains all template DNA strands after cell division. Cell 148, 112-125 (2012).

57. Theret, M. et al. AMPKalpha1-LDH pathway regulates muscle stem cell self-renewal by controlling metabolic homeostasis. EMBO J. 36, 1946-1962 (2017).

58. Barrallo-Gimeno, A. \& Nieto, M. A. The Snail genes as inducers of cell movement and survival: implications in development and cancer. Development 132, 3151-3161 (2005).

59. Conboy, I. M., Conboy, M. J., Smythe, G. M. \& Rando, T. A. Notch-mediated restoration of regenerative potential to aged muscle. Science 302, 1575-1577 (2003).

60. Wen, Y. et al. Constitutive Notch activation upregulates Pax7 and promotes the self-renewal of skeletal muscle satellite cells. Mol. Cell Biol. 32, 2300-2311 (2012).

61. Carlson, M. E. et al. Molecular aging and rejuvenation of human muscle stem cells. EMBO Mol. Med 1, 381-391 (2009).

62. Rodriguez, C. I. et al. High-efficiency deleter mice show that FLPe is an alternative to Cre-loxP. Nat. Genet 25, 139-140 (2000).

63. Sacco, A., Doyonnas, R., Kraft, P., Vitorovic, S. \& Blau, H. M. Self-renewal and expansion of single transplanted muscle stem cells. Nature 456, 502-506 (2008).

64. Kikuchi, M. et al. Development of guided bone regeneration membrane composed of beta-tricalcium phosphate and poly (L-lactide-co-glycolide-coepsilon-caprolactone) composites. Biomaterials 25, 5979-5986 (2004). 
65. Merico, D., Isserlin, R., Stueker, O., Emili, A. \& Bader, G. D. Enrichment map: a network-based method for gene-set enrichment visualization and interpretation. PLoS ONE 5, e13984 (2010).

66. Isserlin, R., Merico, D., Voisin, V. \& Bader, G. D. Enrichment Map-a Cytoscape app to visualize and explore OMICs pathway enrichment results. F1000Res 3, 141 (2014).

\section{Acknowledgements}

We thank Dr. Liangyou Rui at The University of Michigan for his guidance on the generation of Slug conditional mouse line and providing mouse R1 ES cells and pLoxP-2FRT vector. We also thank Dr. Michael Kyba for provision of the Pax7-szGreen mouse strain. This work was funded by NIA R01-AG040182 to W.S.W.

\section{Author contributions}

Conceptualization, P.Z. and W.S.W; Methodology, P.Z., C.P., Y.G., F.W. and Y.Z.; Investigation, P.Z., C.P., Y.G., F.W. and Y.Z.; Formal Analysis, P.Z., C.P., Y.G., F.W and Y.Z.; Writing, P.Z. and W.S.W.; Resources, P.Z., Y.G., F.W. and Y.Z.; Funding Acquisition, W.S.W; Supervision, W.S.W.

\section{Additional information}

Supplementary Information accompanies this paper at https://doi.org/10.1038/s41467019-10479-4

Competing interests: The authors declare no competing interests.
Reprints and permission information is available online at http://npg.nature.com/ reprintsandpermissions/

Journal peer review information: Nature Communications thanks Jody Haigh, Masashi Narita and other anonymous reviewer(s) for their contribution to the peer review of this work. Peer reviewer reports are available.

Publisher's note: Springer Nature remains neutral with regard to jurisdictional claims in published maps and institutional affiliations.

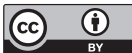

Open Access This article is licensed under a Creative Commons Attribution 4.0 International License, which permits use, sharing, adaptation, distribution and reproduction in any medium or format, as long as you give appropriate credit to the original author(s) and the source, provide a link to the Creative Commons license, and indicate if changes were made. The images or other third party material in this article are included in the article's Creative Commons license, unless indicated otherwise in a credit line to the material. If material is not included in the article's Creative Commons license and your intended use is not permitted by statutory regulation or exceeds the permitted use, you will need to obtain permission directly from the copyright holder. To view a copy of this license, visit http://creativecommons.org/ licenses/by/4.0/.

(C) The Author(s) 2019 